\title{
Facial Expressions of Emotion Categories are Embedded within a Dimensional Space of Valence-arousal
}

Meng Liu ${ }^{1}$, Yaocong Duan ${ }^{1}$, Robin A. A. Ince ${ }^{2}$, Chaona Chen ${ }^{1}$, Oliver G. B. Garrod ${ }^{1}$, Philippe G.

$$
\text { Schyns }^{1,2} \text {, Rachael E. Jack }{ }^{1,2^{*}}
$$

${ }^{1}$ School of Psychology, University of Glasgow, 62 Hillhead Street, Glasgow, Scotland G12 8QB, United Kingdom

${ }^{2}$ Institute of Neuroscience and Psychology, University of Glasgow, 62 Hillhead Street, Scotland G12 8QB, United Kingdom

*Rachael E. Jack

Email: rachael.jack@glasgow.ac.uk

\begin{abstract}
One of the longest standing debates in the emotion sciences is whether emotions are represented as discrete categories such as happy or sad or as continuous fundamental dimensions such as valence and arousal. Theories of communication make specific predictions about the facial expression signals that would represent emotions as either discrete or dimensional messages. Here, we address this debate by testing whether facial expressions of emotion categories are embedded in a dimensional space of affective signals, leading to multiplexed communication of affective information. Using a data-driven method based on human perception, we modelled the facial expressions representing the six classic emotion categories - happy, surprise, fear, disgust, anger and sad - and those representing the dimensions of valence and arousal. We then evaluated their embedding by mapping and validating the facial expressions categories onto the valencearousal space. Results showed that facial expressions of these six classic emotion categories formed dissociable clusters within the valence-arousal space, each located in semantically congruent regions (e.g., happy facial expressions distributed in positively valenced regions). Crucially, we further demonstrated the generalization of the embedding beyond the six classic categories, using a broader set of 19 complex emotion categories (e.g., delighted, fury, and terrified). Together, our results show that facial expressions of emotion categories comprise specific combinations of valence and arousal related face movements, suggesting a multiplexed signalling of categorical and dimensional affective information. Our results unite current theories of emotion representation to form the basis of a new framework of multiplexed communication of affective information.
\end{abstract}




\section{Introduction}

Communicating emotions is essential to human social interaction because it enables predicting the behaviors of others and engaging in adaptive action in response. One of the most effective ways to communicate emotions is by using facial expressions - that is, specific face movement combinations such as eyebrow raising and mouth gaping that others readily perceive as representing certain emotions. For example, broad smiles are often perceived as representing the positive, non-threatening states of happiness or friendliness (1-6), whereas snarls are often perceived as representing the potentially threatening, negative states of anger or disgust. Though emotions are pervasively influential in human life and are communicated regularly to guide everyday interactions, the nature of their representation - that is, their ontology - remains a source of debate. In other words, how are emotions represented by facial expressions?

One influential ontology posits that human emotions, in all their variety, are reducible to a small set of discrete emotion categories, often called 'basic' emotions - namely, happy, surprise, fear, disgust, angry, and sad (e.g., 7-10) - which are each represented by a distinct facial expression (11). For example, that surprise is represented by facial expressions comprising raised eyebrows, wide opened eyes, and a wide opened mouth, whereas anger is represented by facial expressions comprising lowered brows and tightened eyelids and lips (12). This ontology proposes that this small set of discrete emotion categories is irreducible and comprises the basic elements of human emotion; combining them together thus enables the formation of the complex range of emotions, including their facial expressions, that occur throughout social life such as jealousy, disappointment, ecstatic, and so on (e.g., 14, 15).

In contrast, another influential ontology posits that emotions are not truly discrete but are instead structured by a set of underlying dimensions such as valence - comprising very positive to very negative states - and arousal - comprising very excited to very calm states affect - which is known as core affect(16-20). In this ontology, emotions are represented primarily according to specific combinations of these broad dimensions - for example, that happy is represented as a positively valenced state with varying levels of arousal from intensely to mildly happy, whereas anger is represented as a negatively valenced, high-arousal state (18). Therefore, this ontology argues that the building blocks of emotion representation are broad dimensions such as valence and arousal (e.g., see also 13 for the structuring role of affective dimensions).

According to each of these contrasting ontologies, theories of communication make different predictions about the form that facial expression signals would take to represent emotions as discrete categories or as dimensional. Specifically, if emotions are represented as discrete categories, then their facial expressions should comprise distinct signals with minimal overlap in their facial movements across emotion categories. Alternatively, if emotion categories are structured by dimensions of valence and arousal, then their facial expressions should comprise specific combinations of facial movements that represent these broad affective dimensions, to which category-specific facial movements could be added.

Here, we tested an alternative ontology that reconciles these contrasted hypotheses. Specifically, we evaluated whether facial expressions of emotion categories comprise specific combinations of face movements that convey valence and arousal. Fig. 1 schematizes this alternative ontology. 


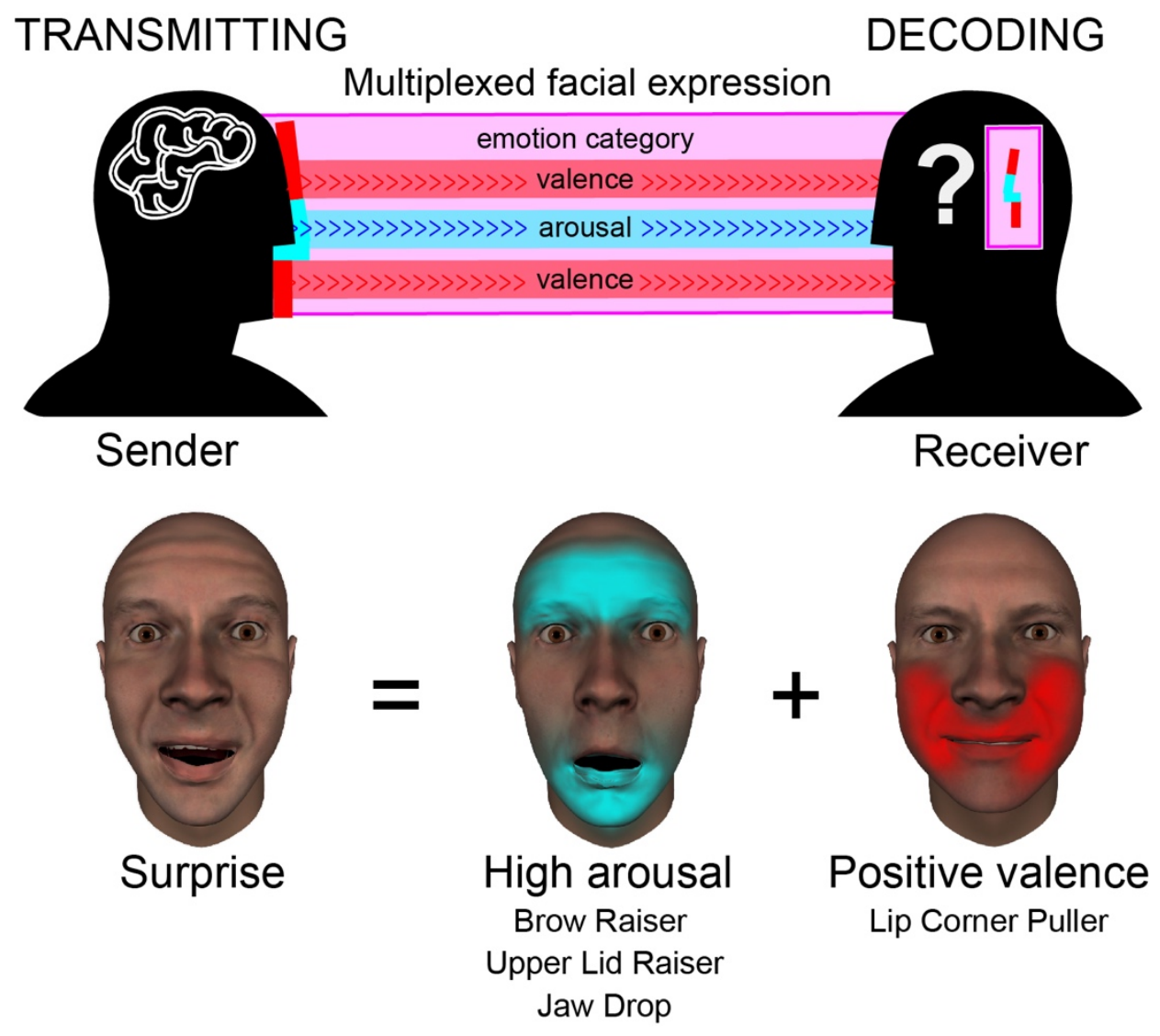

Fig. 1. Multiplexed facial expressions of emotion: a new ontology. To communicate affective messages to others, the sender encodes the message ("I'm surprised") in a signal such as a facial expression that is composed of different individual face movements called Action Units (AUs, 12, 21) - for example, Brow Raiser (AU1-2), Upper Lid Raiser (AU5), Jaw Drop (AU26) and Lip Corner Puller (AU12). In the composition of such a complex signal, the elements of the facial expression could comprise affective dimensions such as valence (positive or negative, represented in red) and arousal (calm or excited, represented in cyan). Such compositions would therefore generate multiplexed facial expression signals where broad affective messages are embedded within the emotion category message.

To test this, we computed the embedding of facial expressions of emotion categories onto a dimensional valence-arousal space of face movements. We proceeded in two steps. First, using a data-driven method, we modelled two sets of facial expressions - (1) those representing the six classic discrete emotion categories - happy, surprise, fear, disgust, anger, and sad - and (2) those representing a dimensional valence-arousal space. We then examined the embedding of the facial expressions of the discrete emotion categories onto the dimensional valence-arousal space. Each step is described in more detail below. 
A. STIMULUS GENERATION

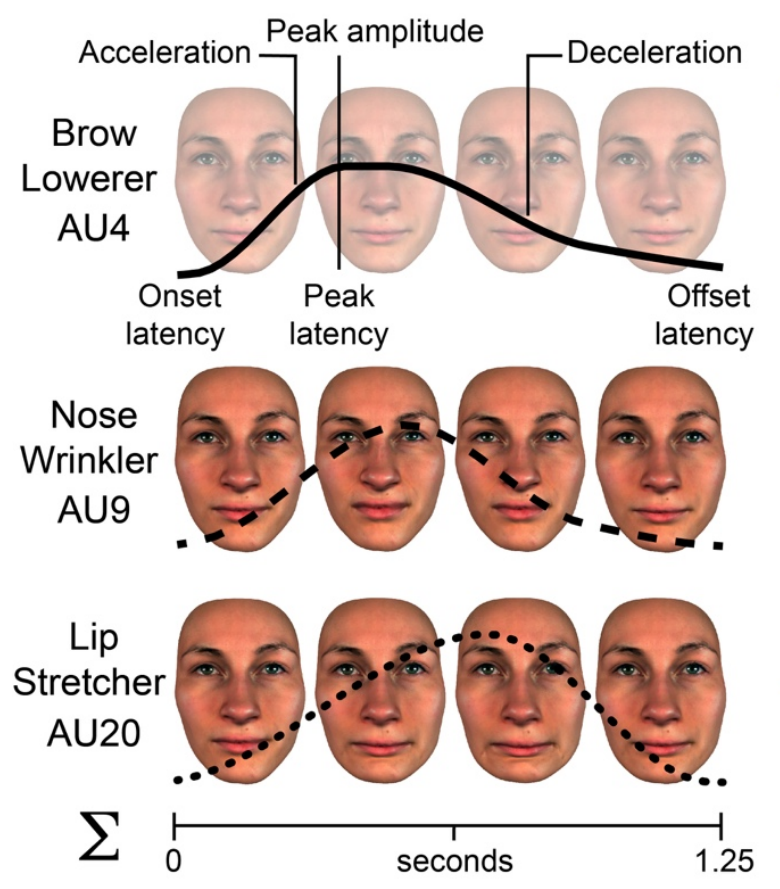

B. PERCEPTUAL TASK

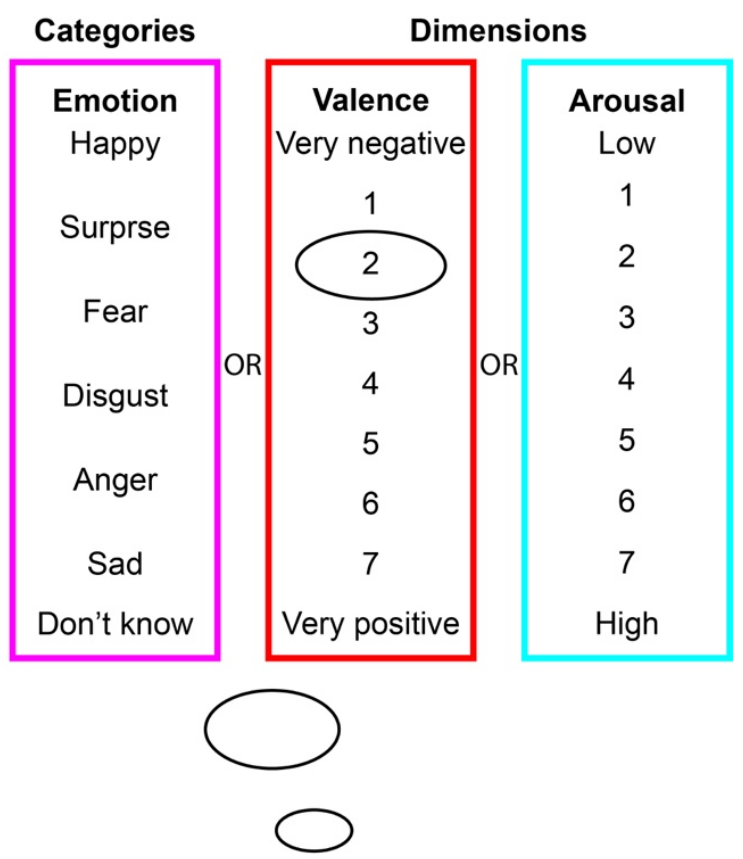

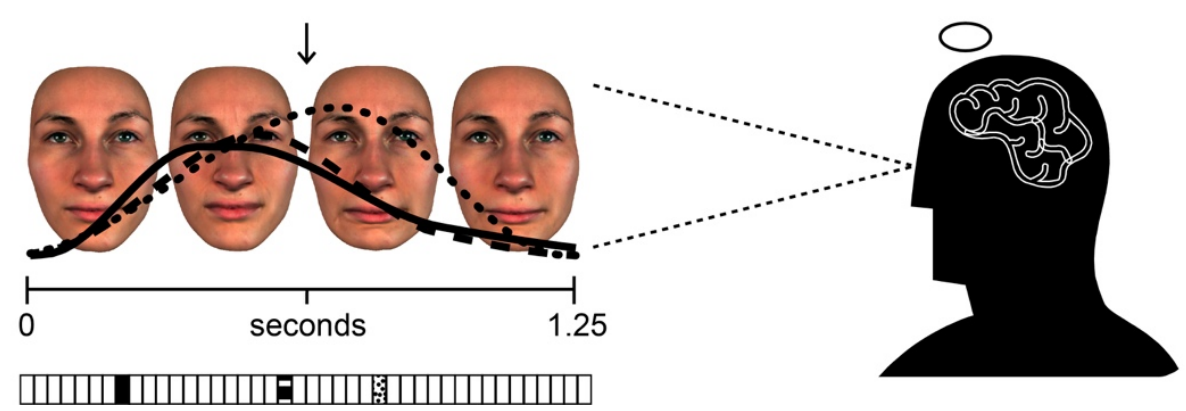

Fig. 2. Stimulus generation and task procedure. (A) Stimulus. On each experimental trial, a face movement generator (22) randomly selected a subset of face movements, called Action Units (AUs, 12) - here, Brow Lowerer (AU4), Nose Wrinkler (AU9), and Lip Stretcher (AU20) - and assigned a random movement to each using six temporal parameters (see labels illustrating the solid curve). These dynamic AUs are then combined to generate a random facial animation, illustrated here with four snapshots across time. The texture-coded vector below represents the 3 (out of 42) AUs randomly selected on this illustrative experimental trial. (B) Perceptual task. Participants viewed the facial animation and interpreted it according to one of three pre-assigned tasks: (1) the six classic emotion categories - happy, surprise, fear, disgust, anger, or sad - or (2) valence - very negative to very positive - or (3) arousal - low arousal to high arousal. In this example trial, the participant interpreted the random facial animation as negative valence. A total of 100 participants (white Western) each completed 2400 such trials in the categorization or dimensional task (see Methods, Participants and Methods, Task Procedure). We displayed all facial animations on one of 8 same-ethnicity face identities (see Methods, Stimuli). 
Data-Driven methodology. To model facial expressions of the six classic emotion categories and a dimensional valence-arousal space, we used a data-driven method that agnostically generates facial expressions and tests them against human perception (23-30). Fig. 2 illustrates the method with an example experimental trial. On each trial, a dynamic face movement generator (22) randomly selects a set of individual face movements called Action Units (AUs, 12) from a core set of 42 AUs (minimum 1 AU, maximum 4 AUs on each trial, median 3 AUs across trials). In this example, three AUs are selected: Brow Lowerer (AU4) represented by the solid curve, Nose Wrinkler (AU9) represented by the dashed curve, and Lip Stretcher (AU20) represented by the dotted curve. Each AU is then assigned a random movement using values randomly selected for each of six temporal parameters - onset latency, acceleration, peak amplitude, peak latency, deceleration, and offset latency (in Fig. 2A, see labels illustrating the solid curve). These randomly generated AUs are then combined to produce a random facial animation, displayed in Fig. 2A as four snapshots across time. The participant views the facial animation and interprets it according to a pre-assigned task. In this example, the participant, assigned to the valence rating task, perceived the facial animation as negatively valenced (see black ellipse). Therefore, each experimental trial captures the face movements that elicit a given perception in the participant for example, here, Brow Lowerer, Nose Wrinkler, and Lip Stretcher, each with a specific dynamic activation, elicited the perception 'medium negative valence.' After many such trials, we can then build the statistical relationship between the dynamic AUs presented on each trial and the participant's responses, thus producing a facial expression model of the specific face movements that the participant associates with a given perception (see Methods, Facial expression modelling procedure). For example, if a participant frequently interpreted facial animations that contain Lip Stretcher (AU20) as 'fear' then this AU would be statistically associated with perceptions of 'fear' in this participant. Each participant completed 2,400 trials within their pre-assigned task with all facial animations displayed on same-ethnicity male and female faces (see Methods, Stimuli).

Modelling of facial expression and their embeddings. We used this method to first build statistically robust facial expression models of (1) the six classic emotion categories for each of 60 individual participants, and (2) a dimensional valence-arousal space using data acquired from a separate group of 40 participants (see Methods, Participants). To evaluate their embedding, we mapped the facial expressions of the six classic emotions onto the dimensional valence-arousal space using cross-correlation and validation (see Results, Embedding facial expressions of emotion categories onto a dimensional valence-arousal space and Fig. 4B). We further replicated the embeddings with an additional set of 19 complex emotion categories, such as delighted, rage, and embarrassed, using an existing dataset (Results, Embedding facial expressions of emotion categories onto a dimensional valence-arousal space, Fig. 4B and SI Appendix Fig. S4). This embedding suggests that facial expressions of emotion categories comprise compositions of valence and arousal face movements. An analysis that decomposed the facial expression of emotion into its constituent face movements revealed this suggested composition (see Results, Composition of facial expressions of emotion categories according to valence and arousal face movements and Fig. 4). Together, our results show that facial expressions of emotion categories are systematically embedded within a dimensional valence-arousal space, suggesting that emotions are represented as multiplexed facial expressions that afford both dimensional and categorical affective communication. Our facial expression models also tease apart the specific dimensional and categorical facial expression contents of these emotion categories. We discuss the implications of our results in uniting existing theories of emotion representation. 


\section{Results}

To evaluate the embedding of facial expressions of emotion categories into a dimensional valence-arousal space, we first used the technique illustrated in Fig. 2 to separately model facial expressions representing (1) the six classic emotion categories and (2) a dimensional space comprising the two dimensions of valence and arousal. Then, we mapped the face movement models of the six emotion categories onto the two-dimensional valence-arousal space and demonstrated that this mapping conforms to the semantic locations of the emotion labels separately obtained. We further validated our theory of multiplexed emotion signaling by repeating these analyses with a broad set of 19 emotion categories and for each (i.e. 6 classic plus 19 complex) computed the multiplexed emotion signal that comprises a valence and arousal component movement plus a residual movement further defining the emotion category. We next describe these steps in turn.

Building facial expression models of the six classic emotion categories. Sixty participants (white Western European, 31 females; see Methods, Participants) each categorized 2,400 randomly generated facial animations according to the six classic emotions of happy, surprise, fear, disgust, anger, or sad, and rated the intensity of each emotion category response on a 5-point scale from 'very weak' to 'very strong.' If none of the six emotion categories matched the facial animation, participants selected 'don't know' (see Methods, Task Procedure). Following the experiment, we built a facial expression model for each of the six emotion categories for each participant. To do so, we measured the relationship between the dynamic AUs presented on each trial and the participant's responses (e.g., happy versus other choices) using nonparametric Mutual Information as a measure of statistical dependence (MI; Ince et al., 2017; see Methods, Facial expression modelling procedure). A high MI value indicates that the AU (e.g., Lip Corner Puller - AU12) is strongly associated with the participant's emotion category response (e.g., 'happy'); a low MI value indicates a weak relationship (e.g. the participants response and the presence of the selected AU are statistically independent). We determined statistical significance using a Monte Carlo permutation method and the method of maximum statistics (Family-Wise Error Rate [FWER] over 42 AUs p < 0.05, see Methods, Facial expression modelling procedure). These AUs are henceforth called "significant AUs." We applied this procedure to the data of each participant, resulting in a total of 360 facial expression models ( 60 participants $\times 6$ emotion categories), each represented as a 42-dimensional binary vector detailing the significant AUs associated with the participant's emotion responses (see $S I$ Appendix, Modelling facial expressions of the six classic emotion categories and Fig. S1). Fig. 3A, Six Classic Emotions, shows the results as face maps representing the significant AUs, summed across participants.

Building facial expression models of a dimensional space of valence and arousal. Next, we built a two-dimensional valence-arousal space of face movements using a similar analysis and a separate group of 40 participants (white Western European, 20 females, see Methods, Participants). Each participant rated a total of 2,400 randomly generated facial animations according to (1) valence on a 7-point scale from 'very 'negative' to 'very positive,' and, in a separate counterbalanced task, (2) arousal on a 7-point scale from 'low arousal' to 'high arousal' (1,200 trials per dimension; see Methods, Task procedure). We then built a facial expression model for each level of valence and each level of arousal (re-scaled to 5 levels to ensure a sufficient number of trials) using a non-parametric statistical method - Pointwise Mutual 
Information (PMI, Bouma, 2009) - and Monte Carlo permutation method to establish univariate statistical significance ( $<<0.05$, see Methods, Facial expression modelling procedure and $S I$ Appendix, Modelling facial expressions of valence and arousal and Fig. S2). These significant AUs therefore formed a $5 \times 5$ dimensional valence-arousal space that cross-combined the significant AUs in a pair-wise manner across the two dimensions (see SI Appendix, Building a valence-arousal space of face movements and Fig. S3). We applied this procedure to the data of each participant. Fig. 3A, Valence-Arousal Space, shows these results as face maps representing the significant AUs, summed across participants. 
A.
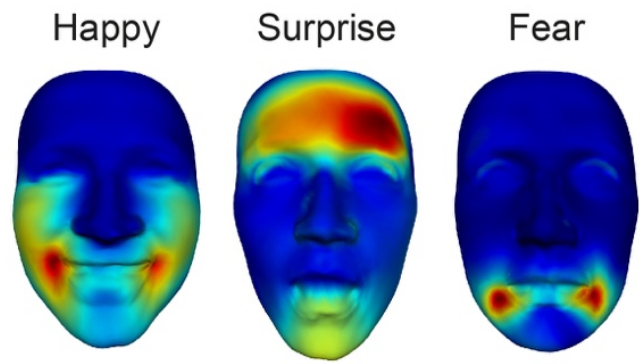

Disgust
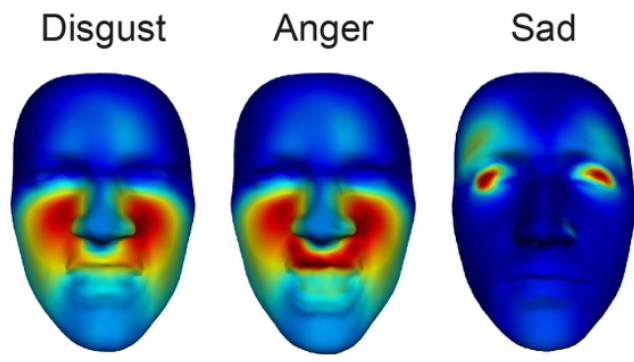

B. PROJECTION PATTERNS
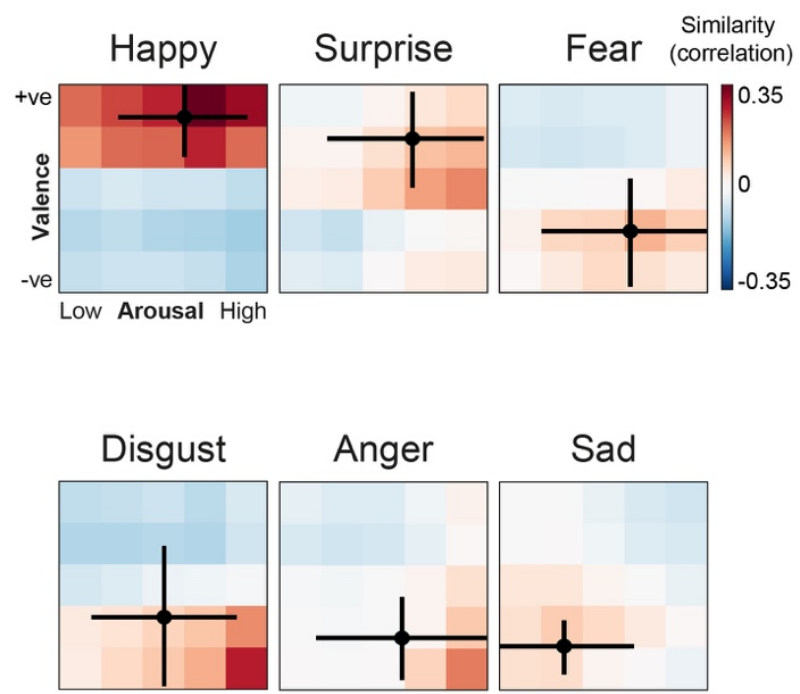

VALENCE-AROUSAL SPACE

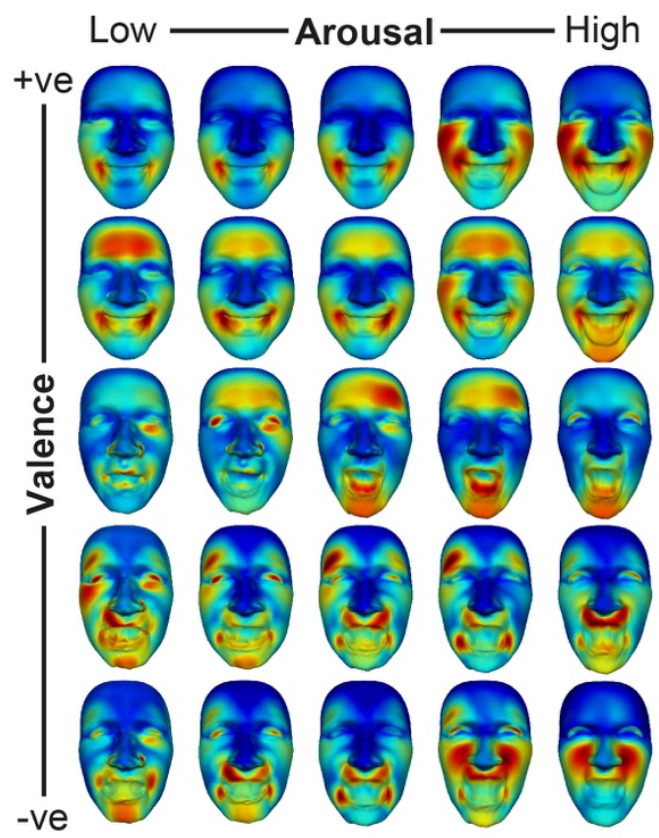

EMBEDDING VALIDATION

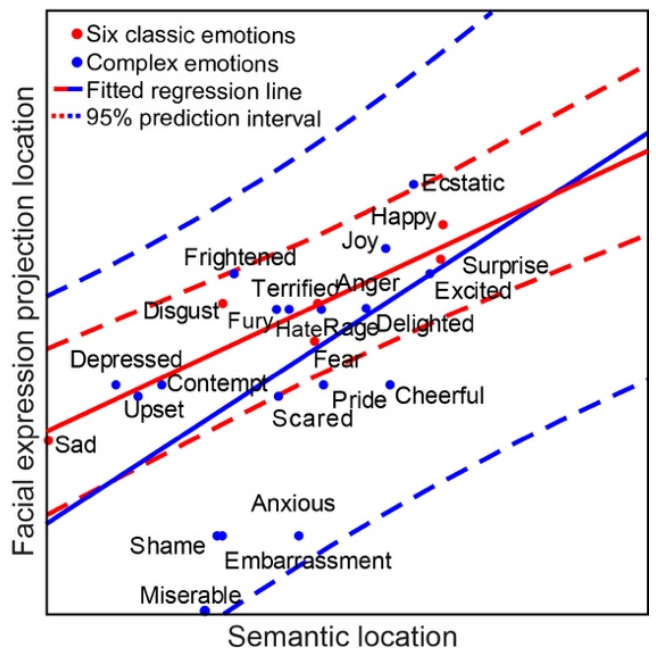

Fig. 3. Mapping facial expressions of emotion categories onto a dimensional valence-arousal space. (A) Facial expressions representing the six classic emotion categories and a dimensional valence-arousal space. Color-coded face maps show the face movements (i.e., Action Units) associated with the six classic emotions (on left) and a dimensional valence-arousal space (on right; see also SI Appendix, Modelling facial expressions of the six classic emotion categories, Fig. S1, and SI Appendix, Building a valence-arousal space of face movements, Fig. S3). (B) Projection patterns of facial expressions of the six classic emotions in valence-arousal space with embedding validation. For each emotion, color-coded matrices show the average correlations 
from the mapping procedure. Dark red indicates high similarity (positive correlation); dark blue indicates high dissimilarity (negative correlation; see color bar on right). For example, happy is positively correlated with positive valence across all levels of arousal; sad is positively correlated with negative valence, low arousal cells. Black crosses show the average (with standard deviation) valence and arousal rating of the emotion word (data extracted from Warriner et al., 2013). We repeated this procedure with a further set of facial expressions of 19 of more complex emotions (see SI Appendix, Mapping facial expressions of complex emotions onto a valencearousal space and Fig. S3). To validate the mappings of all emotions, we regressed the location of the maximum average correlation of the facial expression (represented as dark red squares) with the location of the emotion word in the valence-arousal space (represented as black crosses; see Results, Embedding facial expression of emotion categories onto a dimensional valencearousal space) . Results, shown on the right panel in red for the six classic emotions and blue for the complex emotions, show close correspondence, thereby validating the mappings.

To illustrate how the face movement patterns vary across the valence-arousal space, first consider the four extreme corners. For example, the top right face, which represents the highest positive valence and highest arousal affect, comprises broad, intense smiling that includes Lip Corner Puller-Lips Part (AU12-25), Lip Corner Puller-Cheek Raiser (AU12-6) and Upper Lid Raiser (AU5). By comparison, the top left face, which represents the highest positive valence but lowest arousal affect, comprises lower intensity, calmer smiling that includes fewer, smaller face movements such as Dimpler (AU14) and Lip Corner Puller (AU12). A similar pattern is observed for negative valenced face movements across the dimension of arousal. Specifically, the bottom right face, which represents the highest negative valence and highest arousal affect, comprises large, visually salient threat-based face movements such as Nose Wrinkler (AU9), Mouth Stretch (AU27), Lip Funneler (AU22), and Upper Lid Raiser (AU5). By comparison, the bottom left face, which represents the highest negative valence but lowest arousal affect, comprises smaller but similarly twisting face movements such as Upper Lip Raiser Right (AU10R), Chin Raiser (AU17), Lip Corner Depressor (AU15), Lid Tightener Left (AU7), and Nostril Dilator (AU38), and in contrast to high arousal face movements, Closed Eyes (AU43). Therefore, for both positive and negative valence affects, visual inspection of the face movement patterns across the arousal dimension shows a smooth (left to right in Fig. 3A) evolution of the signal from milder to more extreme face movements. Specifically, as shown across the high arousal faces from positive to negative valence, high arousal affect is characterized by large opening face movements such as the wide stretched mouth and wide opened eyes that shows the eye whites. Such face movements are therefore common across both positive and negative affect face movements and likely serve the role of communicating high arousal states. In contrast, the valence of the signal varies in part with mouth shape, as observed from the face movements across the valence dimension (top to bottom in Fig. 3A) for both high and low arousal - for example, the smiling mouth, which is smooth and flat against the face and associated with positive affect, evolves into more of a twisted, protruding shape for negative affect.

\section{Embedding facial expressions of emotion categories onto a dimensional valence-arousal} space. Six classic emotions. We then tested the embedding of the facial expressions of the six classic emotion categories into the valence-arousal space of face movements. To this aim, we computed, for each of the six emotions separately, the average correlation between the 60 facial expression models of the emotion category and the 40 facial expression models of each cell of the valence-arousal space. Fig. 3B, Projection Patterns, shows these average correlations for each of 
the six emotion categories displayed as color-coded matrices over the valence-arousal space. Reds indicate higher average similarity (i.e., positive correlations) and blues indicate lower average similarity (negative correlations; see colorbar on right). For example, happy facial expressions are positively correlated exclusively with positive valence across all levels of arousal, as shown by the two rows of dark red squares corresponding to higher valence. In contrast, disgust facial expressions are positively correlated exclusively with negative valence and primarily high arousal cells. To validate the embeddings of the facial movements of the six classic emotion categories into the face movements of the valence-arousal space, we checked whether the locations of maximal embedding of the facial movements just reported (e.g. of fear) corresponded (i.e. conforms) to the semantic locations of the valence and arousal of the emotion words (e.g. "fear," as rated by a group of independent participants, data extracted from Warriner, Kuperman, \& Brysbaert, 2013, projected onto a 5-scale). To do so, we linearly regressed the location of the maximum embedding value (i.e. correlation) of each emotion in the valencearousal space (represented in darkest red in Fig. 3B) with the semantic location of the corresponding emotion word in the valence-arousal space (represented as the black cross) based on its average valence and arousal ratings (data extracted from Warriner et al., 2013). We determined the location of each in 2D space by measuring its Euclidean distance from the origin $(0,0)$. Fig. 3B, Embedding Validation, shows these results in red, where the solid line represents the fitted regression and the dashed line represents the $95 \%$ prediction interval $(P=0.0212)$.

Results confirm that the facial expression embeddings conform with the semantic location of their emotion words - for example, facial expressions of fear distribute closely to the semantic location of the emotion word 'fear,' validating our analysis of the embedding of facial movement categories into the facial movements of the valence-arousal space.

Broad set of complex emotions. Next, we evaluated the generalizability of the above embedding of the classic six emotion categories into the valence-arousal space to a more complex set of 19 emotion categories. Using an existing data set collected using the same procedure as illustrated in Fig. 2 and comprising 19 complex emotions such as delighted, rage, and terrified (see Jack et al., 2016), we mapped and validated the facial expressions of each complex emotion onto the valence-arousal space using the same procedure as described above (see SI Appendix, Mapping facial expressions of complex emotions onto a valence-arousal space for full details and Fig. S4 for projection patterns). Fig. 3B, Embedding Validation, shows the results in blue $(P=0.00418)$, confirming validation of the facial expression mappings onto the valence-arousal space.

\section{Composition of facial expressions of emotion categories according to valence and arousal}

face movements. Using the above procedure, we have shown that facial expressions of emotion categories are systematically embedded within a dimensional valence-arousal space of face movements. This suggests that facial expressions of emotion categories embed specific compositions of valence and arousal face movements plus residual emotion movements. To evaluate this multiplexed signal, we decomposed each facial expression model of each of the 25 emotion categories ( 6 classic +19 complex) into its constituent AUs and examined the composition of AUs according to their valence and arousal associations and the residual emotion movements, established using the same PMI analysis described above applied to data pooled across participants (see SI Appendix, Composition of facial expressions of emotion categories and Fig. S5). Fig. 4 shows the results using four illustrative examples (see SI Appendix, Composition of facial expressions of emotion categories and Fig. S6 for full results). 
TRANSMITTING

DECODING

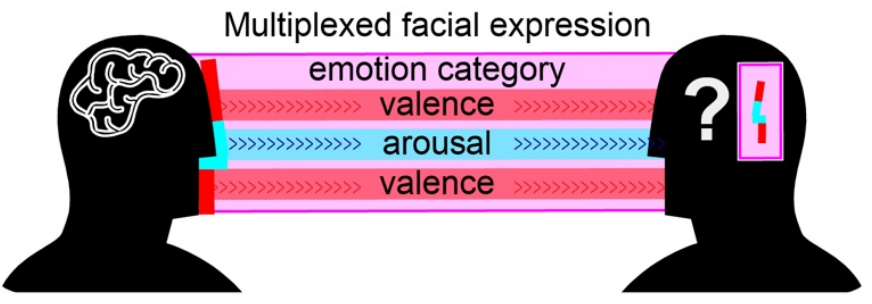

Sender

Receiver

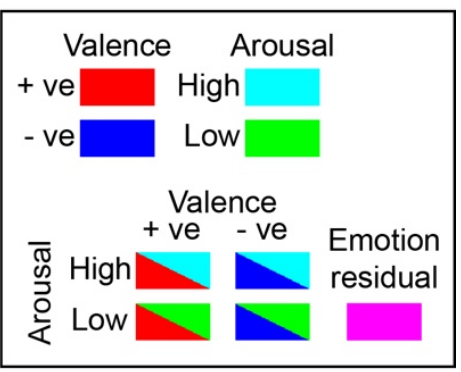

Facial expression
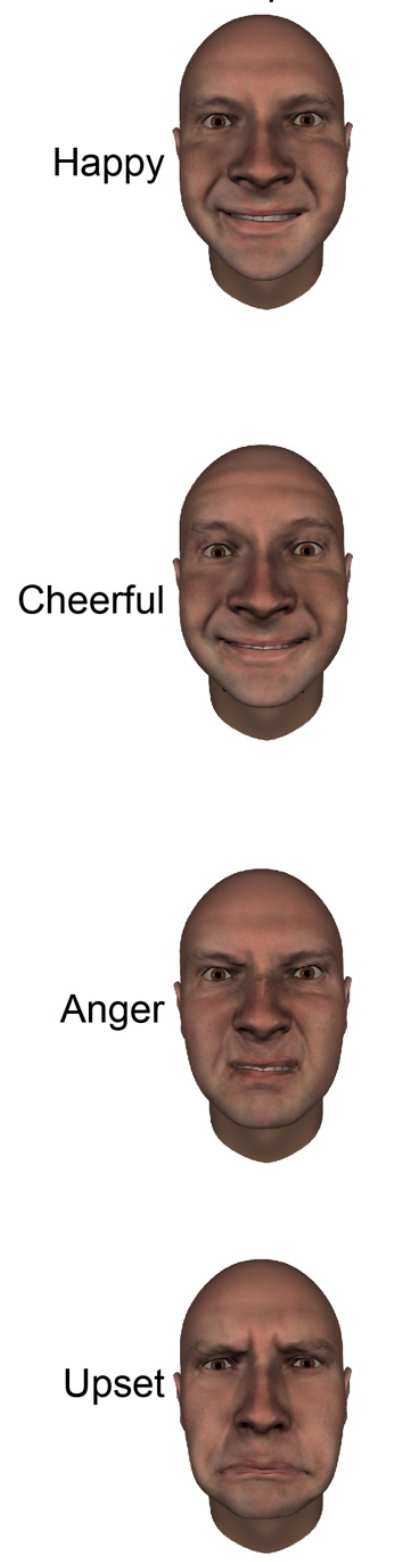

Composition of face movements

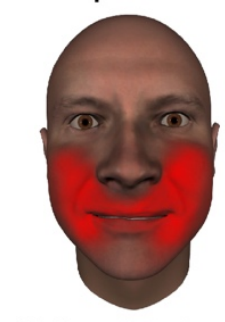

Lip Corner Puller (AU12)
Dimpler R (AU14R)

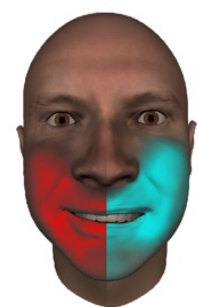

Lip Corner PullerLips Part (AU12-25)

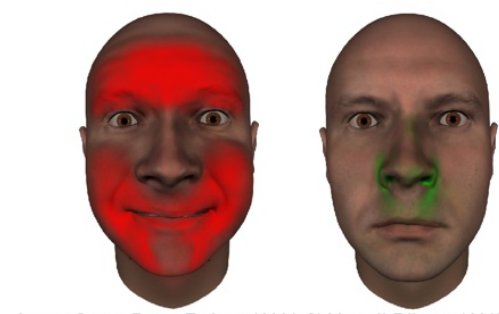

Inner-Outer Brow Raiser (AU1-2) Nostril Dilator (AU38) Outer Brow Raiser (AU2) Lip Corner Puller R (AU12R) Lip Corner Puller (AU12) Sharp Lip Puller (AU13) Lid Tightener L (AU7L) Dim
Lip Corner Puller-Cheek Raiser (AU12-6)

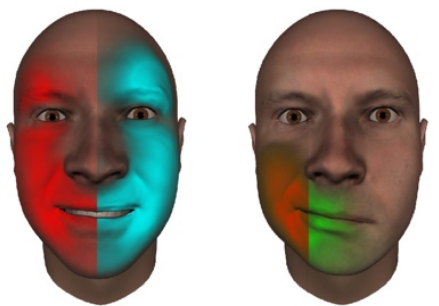

Dimpler R (AU14R)

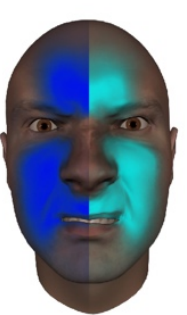

Nose Wrinkler (AU9) Upper Lip Raiser (AU10)

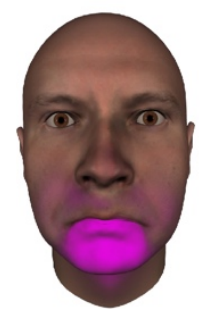

Lip Pressor (AU24)
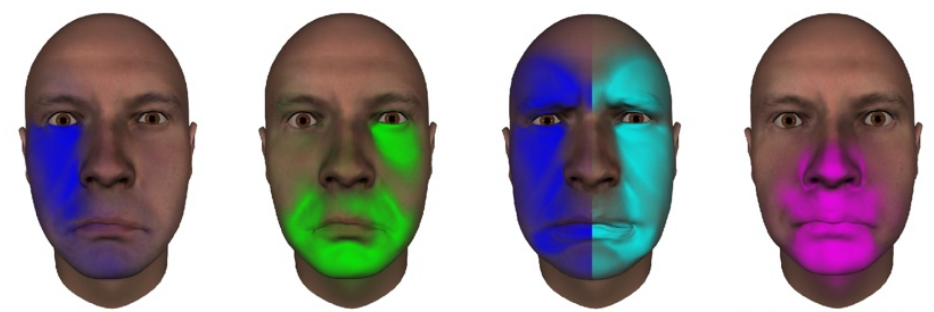

Brow Lowerer (AU4) Lip Pressor (AU24)

) Cheek Raiser (AU6) Nostril Compressor (AU39) Lip Stretcher (AU20) 
Fig. 4 Composition of facial expressions of emotion by valence and arousal face movements. Each row of faces shows the composition of individual face movements of a specific facial expression of emotion - here, we show four illustrative examples, happy, cheerful, anger, and upset. Component face movements are color-coded according to their perceptual association with valence and/or arousal (or neither; see key at top). For example, the facial expression model of anger comprises Nose Wrinkler (AU9) and Upper Lip Raiser (AU10) each of which are associated with high arousal, negative affect, plus Lip Pressor (AU24), which is not associated with valence or arousal but with the perception of the emotion category anger.

In Fig. 4, each row shows a facial expression of emotion (left-most face) decomposed into its constituent face movements and color-coded according to their embedded valence and/or arousal movements (or neither; see key at top). Here, we use four illustrative examples across the range of valence and arousal - happy, cheerful, anger and upset. For example, the facial expression of cheerful comprises a combination of face movements that are associated with positive valence, and elements of both high and low arousal. Fig. S6 shows the full set of results. Here, we show that facial expressions of emotion categories embed specific combinations of face movements that convey broad dimensional information of valence and arousal and for some additional residual movements associated with a specific emotion. Together, our results suggest that facial expressions of emotion are multiplexed signals that afford the communication of both dimensional information and emotion categories.

\section{Discussion}

Here, we have addressed the longstanding debate of whether facial emotions are represented as discrete categories or as dimensions, and proposed that, in contrast to existing ontologies, they do both. Our results show that facial expressions of emotion categories are systematically embedded within a space of valence and arousal and comprise specific combinations of face movements that represent the affective dimensions of valence and arousal. Together, these results suggest that facial expressions of emotions are multiplexed signals that afford the communication of both categorical and dimensional affective information. We demonstrated such multiplexing by examining the correspondence between facial expressions that represent emotion categories and those representing affective dimensions of valence and arousal. First, we used the data-driven method of reverse correlation to build facial expression models of the six classic emotion categories, and a separate dimensional valence-arousal space of face movements. We then mapped the facial expressions of the six classic emotions onto the valence-arousal space using cross-correlation and examined their resulting projection patterns. Results showed systematic embedding of the facial expressions of the six classic emotion categories into the valence-arousal space consistent with purely semantic embedding. We replicated these results with an additional set of facial expressions of 19 more complex emotions including delighted, embarrassed, pride, and rage. In both cases, the embedding of face movements conformed with the independent semantic ratings of the emotion category in the valence and arousal space. Finally, we decomposed facial expressions of the six classic and 19 complex emotions into their constituent face movements and showed that each is primarily composed of a specific combination of valence and arousal face movements. Together, these results suggest that facial expressions of emotion categories are multiplexed signals that afford both categorical and dimensional communication. We now examine the implications of these findings for the representation and decoding of facial expressions of emotion. 
Uniting categorical and dimensional theories of emotion representation. Our results show that facial expressions of emotion categories are composed of specific combinations of face movements that represent broad affective dimensions of valence and arousal. Together, these results provide insights into the nature of emotion representation in terms of discrete categories and dimensions. Before outlining these main insights, it is useful to first consider what is meant by emotions being discrete or dimensional? At one extreme end, a discrete facial expression emotion is one that has no relation to other facial expressions of emotion with no underlying structure (technically, orthogonal in its facial movements to all other facial expressions of emotion). A less extreme view is that emotions are discrete because they have a clear boundary between emotion categories, and thus assumes an underlying structure on which boundaries are placed to demarcate different emotions. For example, by moving from one end of the valence dimension to the other, several emotion categories may be encountered such as ecstatic, delighted, pride, excited, surprise, joy, happy, cheerful, through to sad, depressed, anxious, shame, embarrassed, disgust, contempt, fear, angry, terrified and rage. Boundaries placed along this valence dimension would indicate the point at which one emotion category starts and another ends. In contrast to this hard boundary, another view is that emotions overlap across the dimension - for example, fear might partially overlap with anger because they both comprise negative affect ranging from a mildly negative to highly negative valence. In this case, emotion categories are relatively discrete and comprise fuzzy rather than hard boundaries that lie along specific underlying dimensions. Our results support the latter view - that emotion categories are represented by discrete signals that are systematically arranged by underlying continuous affective dimensions and that have a degree of overlap with other emotion signals. We now unpack out findings in turn.

First, our results show that facial expressions of emotion categories are systematically embedded into the valence-arousal space, which demonstrates their representation as discrete signals $(9,11$, 34). However, their systematic distribution within the valence-arousal space and the composition of their constituent face movements also shows that they comprise combinations of face movements that represent affective dimensional information. For example, facial expressions of disgust distribute in negative, primarily high arousal regions because they comprise combinations of face movements that convey negative valence, high arousal affect such as Nose Wrinkler (AU9) and Lip Stretcher (AU20). These results show that while facial expressions of emotion categories are embedded in distinct regions of the valence-arousal space, they are also systematically structured by broad affective dimensions, thus highlighting the inherent connection between categories and dimensions (e.g., 36). A second feature is that facial expressions of emotion categories show systematic overlap in their embeddings and face movement compositions, which demonstrates that they share certain signaling features such as common face movements and/or underlying affective messages. For example, both fear and disgust are embedded in similar negative valence, high arousal regions on the basis of the common Lip Stretcher (AU20). Therefore, our results show that facial expressions of emotion categories are systematically structured by underlying affective dimensions and comprise fuzzy boundaries, mirror existing work on emotion representation with words (e.g., 13, also see 33 for comments).

Our results also provide further insights into the specific dimensions on which emotion signals vary. Specifically, the embedding of facial expressions of emotion categories in the valencearousal space show they tend to vary in arousal but not valence. For example, facial expressions of happy are embedded across all levels of arousal and exclusively within positive valence cells. This suggests that within an emotion category, arousal is the main dimension of signal variance, whereas valence varies most across emotion categories. A notable exception is surprise, which 
varies across both dimensions of valence and arousal, reflecting its communicative versatility (36). Furthermore, as shown in Fig. 3A, face movements vary across arousal in systematic ways for example, low arousal positive facial expressions comprise small face movements such as closed smiling mouth (Lip Corner Puller - AU12), whereas high arousal positive facial expressions comprise larger facial movements such as broader, open-mouth smile with cheek raising (Lip Corner Puller-Cheek Raiser - AU12-6) and the eye whites (Upper Lid Raiser - AU5). These results suggest that emotion category signals comprise both discrete and continuous elements to convey specific affective information such as high arousal by increasing the amplitude of a smile and/or adding the salient eye whites. This structured variance within emotion categories also aligns with current work showing that emotion categories are bridged by smooth gradients along affective dimensions $(13,37)$. Together, these results further support an ontology where emotions are represented as multiplexed messages of both categorical and dimensional information and further demonstrate the inherent connection between emotion categories and affective dimensions.

Decoding categorical and dimensional information from facial expressions of emotion. Our results suggest that emotions are represented as multiplexed messages comprising both categorical and dimensional information, which raises the question of how such information is processed during decoding. For example, broad dimensional affective information could be processed separately from emotion category information with one informing (i.e. serving as priors for) the other; alternatively, both types of information could be processed simultaneously to arrive at a more refined percept. We showed that the facial expressions that represent one emotion category can transmits variant affective states - for example, anger that is particularly negative - which suggests that dimensional information could be perceived first. Specifically, if observers perceive the emotion category first such as anger and then infer the affective state from this category - for example, negative, high arousal - then we would expect a one-to-one relationship between emotion categories and affective information. That is, the emotion category indicates the specific affective information. However, our data show that emotion categories span variable levels of arousal and, sometimes, valence (e.g., surprise) and thus cannot predict precise levels of valence and arousal. On the other hand, a given level of valence and arousal can predict with high accuracy an emotion category because emotion categories are distributed in distinct multivariate patterns. Even though there are face movement in emotion categories are not covered by combined dimensional information, we can test whether this missed face movements are critical for the human to perceive the facial expression as such emotion.

Evidence of a multiplexed message raises the question of the synchronous, or asynchronous nature of its decoding in the brain. Though we did not concurrently measure the brain activity of participants resolving these affect perception tasks, we can lay out predictions for such future work. The asynchronous account predicts that dimensional information could be processed first, either because it is more robust to degradation in the communication channel due to changes in viewing distance, for example (see 38), or because it serves to narrow down the number of possible emotion categories in a two stage process from broad to specific processing (see also 41). For example, the emotion category of anger with a particularly high level of arousal, as signalled by specific face movements such as the eye whites (see 39, 40). The synchronous account suggests that both categorical and dimensional information are processed within the same time window rather than in a specific temporal structure, with each providing unique information. Exploring these possibilities with methodologies that trace the time course of 
representations of face movements and their semantics (42-44) will be the focus of future research.

\section{Conclusions}

Here, we found that facial expressions of emotion categories are structured by an underlying set of face movements that convey broad dimensions of valence and arousal, as is observed with emotion concepts $(13,45)$, attitudes (e.g., 45), well-being (e.g., 46), attention (e.g., 47), and memory (e.g., 48). Our results address longstanding questions about the ontology of emotion representation by showing that emotions are represented as multiplexed messages comprising both categorical and dimensional affective information. Our results therefore unite current theories of emotion and form the basis of a new framework that reflects the complexities of emotion representation. Our results also highlight the strength of using data-driven methods and robust statistical tools advance understanding of the complexities of facial expression communication. We anticipate that our work and approach will generate a richer and more accurate account of emotion communication.

\section{Methods}

Participants. For the emotion categorization task, we used an existing data set comprising 60 participants (white Western European, 31 females, mean age $=22$ years, $S D=1.71$ years; see Jack et al., 2014) and recruited a separate set of 40 participants white Western European, 20 females, mean age $=21$ years, $S D=2.52$ years) for the dimensional rating tasks of valence and arousal. All 100 participants had minimal experience of/exposure to non-Western cultures (as assessed by questionnaire, see SI Appendix - Screening Questionnaire, e.g., see De Leersnyder et al., 2011), normal or corrected-to-normal vision, and free from any emotion-related atypicalities (Autism Spectrum Disorder, depression, anxiety), learning difficulties (e.g., dyslexia), synaesthesia, and disorders of face perception (e.g., prosopagnosia) as per self-report. All participants gave written informed consent prior to testing and received a standard rate of $£ 6 / \mathrm{h}$ for their participation. The University of Glasgow College of Science and Engineering Ethics Committee provided ethical approval (Ref: 300180112).

Stimuli. For the emotion categorization task, as described in Jack et al., (2014) and illustrated in Fig. 2, we generated 2400 random facial animations and displayed each on one of 8 photorealistic face identities (white Western European, 4 female, mean age $=28$ years, $S D=3.85$ years) captured using a high-resolution 3D face capture system (see Yu, Garrod, \& Schyns, 2012 for full details). For the dimensional valence and arousal rating tasks, we generated a further 2400 random facial animations and displayed each on the same set of 8 face identities as in the emotion categorization task. For each participant in the dimensional rating task, we randomly split these stimuli into two sets of 1200 facial animations and assigned each set to one of the two tasks of valence and arousal.

Task procedure. As illustrated in Fig. 2, participants viewed a randomly generated facial animation on each trial and interpreted it according to one of three pre-assigned tasks - (1) six classic emotion categories, (2) ratings of valence or (3) ratings of arousal. Each facial animation played once for a duration of 1.25 seconds followed by a black screen. Participants responded 
using a Graphic User Interface (GUI) with unlimited time to respond. The next trial started after the participant responded to the preceding trial. We displayed all facial animations on a black background on a 19-inch flat panel Dell monitor (Round Rock, Texas 78682, refresh rate of 60 $\mathrm{Hz}$ and resolution of $1024 \times 1280$ ) in the centre of the participant's visual field. Participants used a chin rest to maintain a constant viewing distance, with stimuli subtending $14.25^{\circ}$ (vertical) $\times$ $10.08^{\circ}$ (horizontal) of visual angle in the emotion categorization task (average stimulus size 17.00 $\mathrm{cm} \times 11.99 \mathrm{~cm}$ ) and $13.74^{\circ} \times 8.65^{\circ}$ of visual angle in the dimensional rating task (average stimulus size $19.54 \mathrm{~cm} \times 12.28 \mathrm{~cm}$ ), each reflecting the average size of a human face during typical social interaction (50). For the dimensional rating task, we blocked the two tasks of valence and arousal, counterbalanced the order of the blocks across participants, and randomized the order of the trials within each block for each participant. For the emotion categorization task, we randomized the order of the trials across the experiment for each participant. For the all three tasks, we divided the trials into separate sessions of 200 trials, each split into 4 sets of 50 trials each separated by a short break. After three consecutive sessions of 200 trials, participants took a required break of at least 1 hour.

\section{Facial expression modelling procedure}

Six classic emotions. To identify the AUs that elicit the perception of the six classic emotions, we used the non-parametric statistical method of Mutual Information (MI, Ince et al., 2017), which measures the statistical dependence between two variables without any assumptions on the nature of the relationship. For each of the six emotions, we computed MI between the AU (present or absent on each trial) and the participants responses with one vs rest coding for the considered emotion, (i.e., "happy" vs "not happy"). A high MI value indicates that the AU is strongly associated with - i.e., predicts - the participant's response; a low MI value indicates a weak relationship. To determine statistical significance, we implemented a non-parametric permutation test by randomly shuffle the participant's responses before re-calculating the MI value for each AU. We repeated this procedure for 1,000 iterations and take the max MI values across all 42 AUs in each iteration to derive a distribution of maximum MI under the null hypothesis that the AU presentation is independent of the participants responses. We rejected this hypothesis for AUs with MI values above the $95^{\text {th }}$ percentile of the max MI distributions. (Family-Wise Error Rate [FWER] over 42 AUs, $P<0.05$ ). We call this method maximum statistics to correct for multiple comparisons. This procedure, applied to the data of each participant, resulted in a total of 360 facial expression models (60 participants X 6 emotion categories), each represented as a 42 dimensional vector detailing the significant AUs associated with the participant's perception of each emotion. Fig. S1 shows the results.

Dimensional valence-arousal space. To identify the AUs that elicit the perception of each level of valence and arousal, we used Pointwise Mutual Information (PMI, Bouma, 2009), which indicates the contribution of each possible pair of values (i.e. AU present and a specific response rating level) to the overall MI value. Firstly, we tested the relationship between AUs and tasks by determining statistical significance MI between each AU and each dimension using a Monte Carlo permutation method and the method of maximum statistics (Family-Wise Error Rate [FWER] over 42 AUs, $P<0.05$, see Methods, Facial expression modelling procedure). Then, we focus on PMI values for the present event of AUs with significant high MI and obtain a PMI value for AU present and for each possible response rating. Then, a positive PMI value indicates that the presence of that AU (e.g., Upper Lid Raiser - AU5) increases the probability of observing that specific rating response (e.g., 'high arousal'). A negative PMI value indicates that the presence of that $\mathrm{AU}$ decreases the probability of observing the considered rating response (e.g., Lip Corner Puller-Cheek Raiser - AU12-6 and 'very negative' valence). We established 
statistical significance of PMI using the same permutation test approach described above and a two-tailed test to identify the AUs that are associated, positively (above $97.5^{\text {th }}$ percentile) or negatively (below $2.5^{\text {th }}$ percentile; $P<.05$ ), with each level of valence and arousal for each participant. We used participant ratings re-scaled from seven to five levels (by iteratively merging the smallest neighboring ratings) to improve sampling and account for the fact that different participants preferred different regions of the response rating range. This procedure resulted in a total of 400 facial expression models ( 40 participants X 2 valence and arousal tasks X 5 levels of rating; see SI Appendix, Modelling face movements of valence and arousal, Fig. S2 for results summed across participants). Finally, we created a 5 X 5 valence-arousal space of face movements by cross-combining in a pair-wise manner the positively associated AUs across the two dimensions and removing any negatively associated AUs (see SI Appendix, Building a valence-arousal space of face movements and Fig. S3 for an illustration). We applied this procedure to the data of each participant, resulting in a total of 40 such 5 X 5 valence-arousal face movement spaces (see SI Appendix, Building a valence-arousal space of face movements and Fig. $\mathrm{S} 3$ for results, represented as face maps in each cell, summed across participants and normalized per cell for visualization purposes). To interpret the composition of facial expressions according to their constituent valence and arousal AUs (see Fig. 4 and Fig. S6 for full results) with a good generalization, we re-computed the PMI between each AU and each level of valence and each level of arousal using the same method described above, applied to data pooled across participants. We determined statistical significance using a Monte Carlo permutation method and the method of maximum statistics (Family-Wise Error Rate [FWER] over 42 AUs, $P<0.05$, see Methods, Facial expression modelling procedure). This method provides a clear categorization of AUs for their contribution to each dimension (see SI Appendix, Facial expression of emotion decomposition and Fig. S5).

\section{Acknowledgments}

This work was supported by the China Scholarship Council [CSC; 101706070134] awarded to ML; the China Scholarship Council [CSC; 201306270029] awarded to CC; the Wellcome Trust [214120/Z/18/Z] awarded to RAAI; the European Research Council [FACESYNTAX - 75858], British Academy [SG113332 and SG171783], Economic and Social Research Council [ES/K001973/1 and ES/ K00607X/1], and John Robertson Bequest [University of Glasgow] awarded to REJ; the Wellcome Trust [Senior Investigator Award, UK; 107802] and the Multidisciplinary University Research Initiative/Engineering and Physical Sciences Research Council [USA, UK; 172046-01] awarded to PGS.

\section{References}

1. P. Ekman, W. V. Friesen, Felt, false, and miserable smiles. J. Nonverbal Behav. 6, 238-252 (1982).

2. U. Hess, M. G. Beaupré, N. Cheung, Who to whom and why-cultural differences and similarities in the function of smiles. Empir. Reflect. Smile 4, 187 (2002).

3. M. Maringer, E. G. Krumhuber, A. H. Fischer, P. M. Niedenthal, Beyond smile dynamics: mimicry and beliefs in judgments of smiles. Emotion 11, 181 (2011).

4. P. M. Niedenthal, J. B. H. \& M. B. Setterlund, Being Happy and Seeing "Happy' ': Emotional State Mediates Visual Word Recognition. Cogn. Emot. 11, 403-432 (1997). 
5. I. Poggi, R. Chrico, The meanings of smile. Oralite Gestualite Commun. Multimodale Interact. Paris Harmattan, 159-164 (1998).

6. M. Rychlowska, et al., Functional smiles: Tools for love, sympathy, and war. Psychol. Sci. 28, 1259-1270 (2017).

7. P. Ekman, Cross-cultural studies of facial expression. Darwin Facial Expr. Century Res. Rev. 169222 (1973).

8. P. Ekman, D. Cordaro, What is Meant by Calling Emotions Basic. Emot. Rev. 3, 364-370 (2011).

9. C. E. Izard, Basic emotions, relations among emotions, and emotion-cognition relations. Psychol. Rev. 99, 561-565 (1992).

10. A. Ortony, T. J. Turner, What's basic about basic emotions? Psychol. Rev. 97, 315 (1990).

11. P. Ekman, An argument for basic emotions. Cogn. Emot. 6, 169-200 (1992).

12. P. Ekman, W. V. Friesen, Facial action coding system: Investigator's guide (Consulting Psychologists Press, 1978).

13. A. S. Cowen, D. Keltner, Self-Report Captures 27 Distinct Categories of Emotion Bridged by Continuous Gradients. Proc. Natl. Acad. Sci. 114, E7900-E7909 (2017).

14. S. Du, Y. Tao, A. M. Martinez, Compound facial expressions of emotion. Proc. Natl. Acad. Sci. 111, E1454-E1462 (2014).

15. K. Oatley, P. N. Johnson-Laird, Towards a cognitive theory of emotions. Cogn. Emot. 1, 29-50 (1987).

16. A. Mehrabian, Pleasure-arousal-dominance: A general framework for describing and measuring individual differences in Temperament. Curr. Psychol. 14, 261-292 (1996).

17. L. F. Barrett, J. A. Russell, The Structure of Current Affect: Controversies and Emerging Consensus. Curr. Dir. Psychol. Sci. 8, 10-14 (1999).

18. J. A. Russell, A Circumplex Model of Affect. J. Pers. Soc. Psychol. 39, 1161-1178 (1980).

19. L. F. Barrett, E. Bliss-Moreau, S. L. Duncan, S. L. Rauch, C. I. Wright, The amygdala and the experience of affect. Soc. Cogn. Affect. Neurosci. 2, 73-83 (2007).

20. S. Schachter, "The Interaction of Cognitive and Physiological Determinants of Emotional State11Much of the research described in this paper was supported by Grant MH 05203 from the National Institute of Mental Health, United States Public Health Service, and by Grant G 23758 from the National Science Foundation." in Advances in Experimental Social Psychology, L. Berkowitz, Ed. (Academic Press, 1964), pp. 49-80.

21. P. Ekman, W. V. Friesen, J. C. Hager, Facial action coding system: The manual on CD ROM. Hum. Face Salt Lake City, 77-254 (2002). 
22. H. Yu, O. G. B. Garrod, P. G. Schyns, Perception-driven facial expression synthesis. Comput. Graph. 36, 152-162 (2012).

23. A. Ahumada, J. Lovell, Stimulus features in signal detection. J. Acoust. Soc. Am. 49, 1751$1756(1971)$.

24. F. Gosselin, P. G. Schyns, Bubbles: a technique to reveal the use of information in recognition tasks. Vision Res. 41, 2261-2271 (2001).

25. R. E. Jack, O. G. B. Garrod, H. Yu, R. Caldara, P. G. Schyns, Facial expressions of emotion are not culturally universal. Proc. Natl. Acad. Sci. 109, 7241-7244 (2012).

26. M. C. Mangini, I. Biederman, Making the ineffable explicit: estimating the information employed for face classifications. Cogn. Sci. 28, 209-226 (2004).

27. P. G. Schyns, L. Bonnar, F. Gosselin, Show Me the Features! Understanding Recognition From the Use of Visual Information. Psychol. Sci. 13, 402-409 (2002).

28. R. E. Jack, P. G. Schyns, Toward a Social Psychophysics of Face Communication. Annu. Rev. Psychol. 68, 269-297 (2017).

29. A. Todorov, C. Y. Olivola, R. Dotsch, P. Mende-Siedlecki, Social Attributions from Faces: Determinants, Consequences, Accuracy, and Functional Significance. Annu. Rev. Psychol. 66, 519-545 (2015).

30. R. Adolphs, L. Nummenmaa, A. Todorov, J. V. Haxby, Data-driven approaches in the investigation of social perception. Phil Trans R Soc B 371, 20150367 (2016).

31. G. Bouma, Normalized ( Pointwise ) Mutual Information in Collocation Extraction in (2009).

32. A. B. Warriner, V. Kuperman, M. Brysbaert, Norms of valence, arousal, and dominance for 13,915 English lemmas. Behav. Res. Methods 45, 1191-1207 (2013).

33. R. E. Jack, W. Sun, I. Delis, O. G. B. Garrod, P. G. Schyns, Four not six: Revealing culturally common facial expressions of emotion. J. Exp. Psychol. Gen. 145, 708-730 (2016).

34. C. G. Kohler, et al., Differences in facial expressions of four universal emotions. Psychiatry Res. 128, 235-244 (2004).

35. J. A. Russell, Facial expressions of emotion: What lies beyond minimal universality? Psychol. Bull. 118, 379 (1995).

36. M. J. Kim, et al., Human amygdala tracks a feature-based valence signal embedded within the facial expression of surprise. J. Neurosci. 37, 9510-9518 (2017).

37. L. F. Barrett, Z. Khan, J. Dy, D. Brooks, Nature of Emotion Categories: Comment on Cowen and Keltner. Trends Cogn. Sci. 22, 97-99 (2018). 
38. F. W. Smith, P. G. Schyns, Smile Through Your Fear and Sadness: Transmitting and Identifying Facial Expression Signals Over a Range of Viewing Distances. Psychol. Sci. 20, 1202-1208 (2009).

39. R. E. Jack, O. G. B. Garrod, P. G. Schyns, Dynamic Facial Expressions of Emotion Transmit an Evolving Hierarchy of Signals over Time. Curr. Biol. 24, 187-192 (2014).

40. C. Chen, et al., Dynamic facial expressions of emotion decouple emotion category and intensity information over time (2020) https:/doi.org/10/gg58gr.

41. B. Giordano, et al., From categories to dimensions: spatio-temporal dynamics of the cerebral representations of emotion in voice (2020).

42. P. G. Schyns, L. S. Petro, M. L. Smith, Dynamics of Visual Information Integration in the Brain for Categorizing Facial Expressions. Curr. Biol. 17, 1580-1585 (2007).

43. P. G. Schyns, J. Zhan, R. E. Jack, R. A. A. Ince, Revealing the information contents of memory within the stimulus information representation framework. Philos. Trans. R. Soc. B Biol. Sci. 375, 20190705 (2020).

44. J. Zhan, R. A. A. Ince, N. van Rijsbergen, P. G. Schyns, Dynamic Construction of Reduced Representations in the Brain for Perceptual Decision Behavior. Curr. Biol. 29, 319-326.e4 (2019).

45. K. R. Scherer, What are emotions? And how can they be measured? Soc. Sci. Inf. 44, 695729 (2005).

46. T. A. Ito, J. T. Cacioppo, Affect and attitudes: A social neuroscience approach. Handb. Affect Soc. Cogn., 50-74 (2001).

47. R. J. Davidson, Affective style, psychopathology, and resilience: brain mechanisms and plasticity. Am. Psychol. 55, 1196 (2000).

48. R. D. Lane, P. M. Chua, R. J. Dolan, Common effects of emotional valence, arousal and attention on neural activation during visual processing of pictures. Neuropsychologia 37, 989-997 (1999).

49. E. A. Kensinger, S. Corkin, Two routes to emotional memory: Distinct neural processes for valence and arousal. Proc. Natl. Acad. Sci. 101, 3310-3315 (2004).

50. E. T. Hall, The hidden dimension (Garden City, NY: Doubleday, 1966).

51. R. A. A. Ince, et al., A statistical framework for neuroimaging data analysis based on mutual information estimated via a gaussian copula. Hum. Brain Mapp. 38, 1541-1573 (2017).

52. J. Dunn, O. J. Dunn, Multiple Comparisons Among Means. Am. Stat. Assoc., 52-64 (1961). 


\section{Supplementary Information}

Modelling facial expressions of the six classic emotion categories. To identify the AUs that are significantly associated with each of the six classic emotion categories, we used Mutual Information (MI; Ince et al., 2017) and a non-parametric permutation test to establish statistical significance. Fig. S1 shows the results, summed across participants, as a color-coded matrix plus corresponding face maps below. Warmer colors indicate higher numbers of participants, cooler colors indicate lower numbers (see colorbar to right).

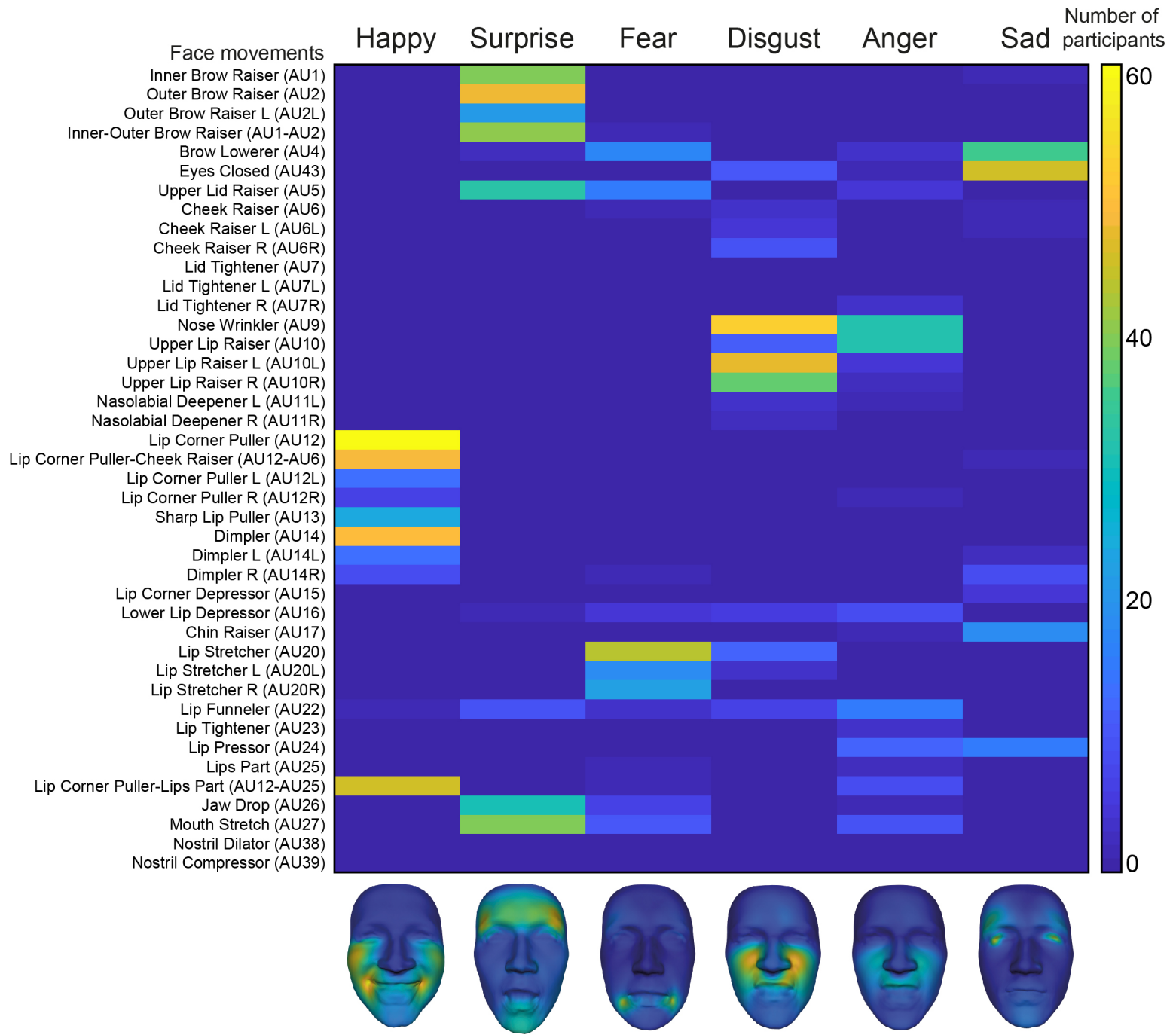

Fig. S1. Face movements representing the six classic emotion categories. The color-coded matrix shows the face movements (i.e., Action Units) that are significantly associated with the perception of each of the six classic emotion categories as determined using Mutual Information 
(Ince et al., 2017). Warmer colors indicate higher numbers of participants, cooler colors lower numbers (see colorbar to right). For example, all 60 participants associated Lip Corner Puller (AU12) exclusively with the emotion category happy. Similarly, most participants associate Upper Lip Raiser Left (AU10) almost exclusively with disgust. Corresponding face maps are shown below.

For example, most participants exclusively associated the emotion category surprise with Outer Brow Raiser (AU2); in contrast, most participants associated both anger and disgust with a common face movement - Nose Wrinkler (AU9). Existing work and a visual inspection of these facial expression patterns show that they comprise dissociable signals (see Jack et al., 2012) with some shared Action Units across emotions such as Upper Lid Raiser (AU5) in fear and surprise.

Modelling facial expressions of valence and arousal. To identify the AUs that are significantly associated with each level of valence and arousal, we used Pointwise Mutual Information (PMI, Bouma, 2009) and a non-parametric permutation test to establish statistical significance. Focusing on AU present events only, we used a two-tailed test to identify the AUs that are associated with each level of valence and arousal, either positively (above $97.5^{\text {th }}$ percentile) or negatively (below $2.5^{\text {th }}$ percentile; $P<.05$ ), for each participant. Fig. S2 shows the results, summed across participants, as color-coded matrices with corresponding face maps below. Warmer colors indicate higher number of participants, cooler colors indicate lower number (see colorbar on right). Panel A shows the positively associated AUs (i.e., when the AU is present, the participant is more likely than chance to choose this response option); panel B shows the negatively associated AUs (i.e., when the AU is present, the participant is less likely than chance to choose this response option). Here chance means there is no relationship between the presenting of AUs and the chosen of response options. 
A.

\section{Positively Associated Face Movements Across Dimensions of Valence and Arousal}

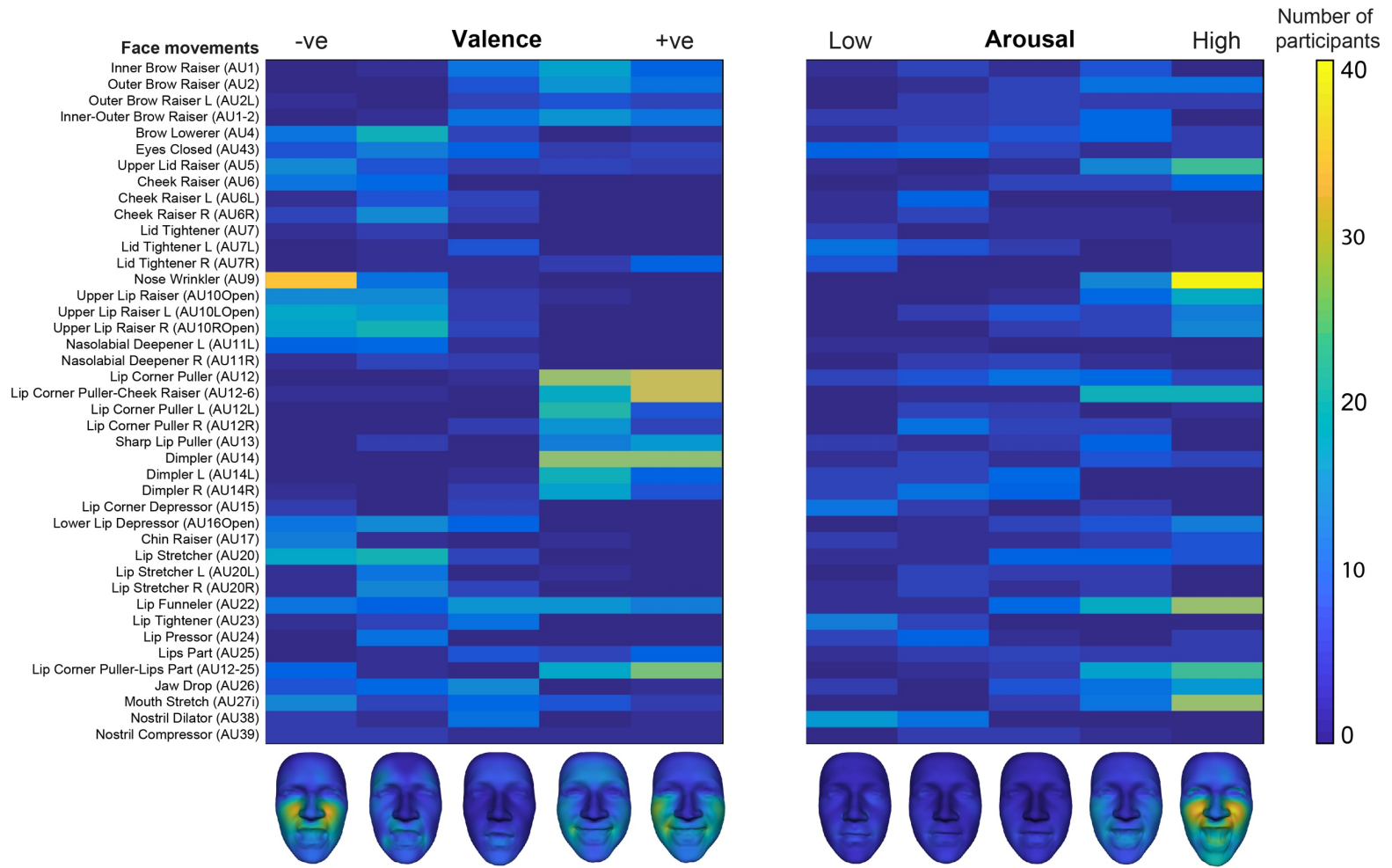

B. Negatively Associated Face Movements Across Dimensions of Valence and Arousal

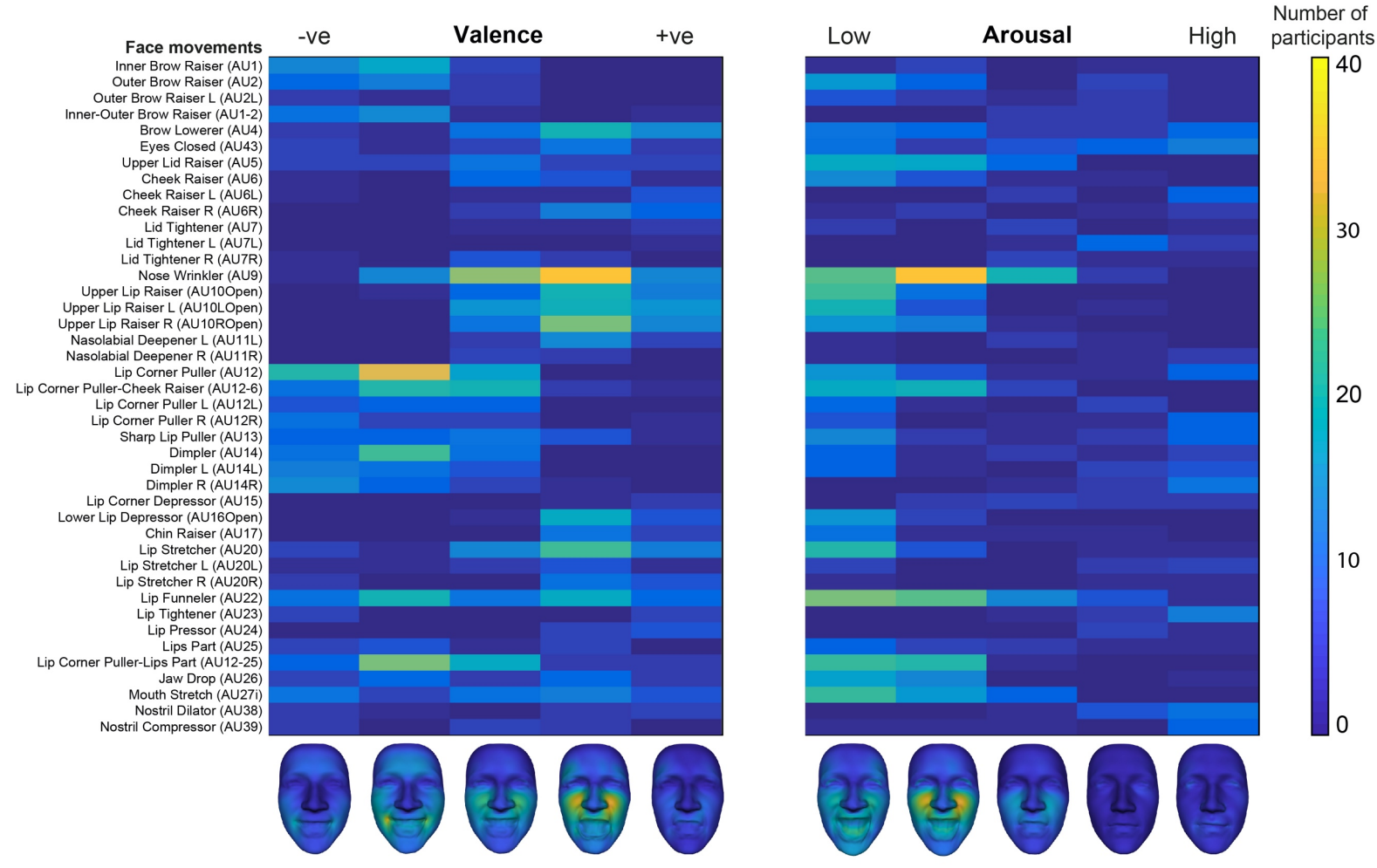


Fig. S2. Face movements associated with the perception of valence and arousal. $(A)$ Positively associated face movements across dimensions of valence and arousal. Each color-coded matrix shows the Action Units (see labels on left) that are significantly positively associated with perceptions of each level of valence (left panel, negative to positive) and arousal (right panel, low to high) - i.e., when the AU is present, the participant is more likely than chance to choose this response option. Warmer colors indicate higher numbers of participants, cooler colors indicate lower numbers (see color bar on right). For example, in valence, Nose Wrinkler (AU9) is positively associated with negative valence in most participants. Corresponding face maps are shown below. (B) Negatively associated face movements across dimensions of valence and arousal. As in Panel A, the color-coded matrix shows the AUs that are negatively associated with perception of different levels of valence and arousal - i.e., when the AU is present, the participant is less likely than chance to choose this response option. For example, Nose Wrinkler (AU9) is negatively associated (i.e., disassociated) with perceptions of positive valence and low arousal in most participants. Corresponding face maps are shown below.

Building a valence-arousal space of face movements. To create a valence-arousal space of face movements, we combined in a pairwise manner the AUs that are positively associated with each level of valence and arousal for each cell of the 5 X 5 space and removed any negatively associated AUs. Fig. S3 illustrates the procedure. For example, to build the face movements that represent medium positive valence (rating 4), high arousal (rating 5) - see in panel A, face map outlined in green - we combined the corresponding positively associated face movements - i.e., medium positive valence (rating 4) and high arousal (rating 5), both outlined in dashed red - and removed any negatively associated AUs - i.e., medium positive valence (rating 4) and high arousal (rating 5), both outlined in dashed blue. Additionally, while most AUs are significantly associated with either valence or arousal, some AUs are associated with both valence and arousal - for example, Nose Wrinkler is positively associated with both negative valence and high arousal. We therefore restricted these AUs to the corresponding specific sub-set of cross-combined cells - e.g., Nose Wrinkler only appears in negative valence, high arousal cells. We applied this procedure to the data from each participant, resulting in a total of 40 such 5 X 5 valence-arousal face movement spaces. Fig S3A shows the resulting 5 x 5 valence-arousal space of face movements. Fig. 3B illustrates this procedure using the same example. 
A. Dimensional Valence-Arousal Space of Face Movements

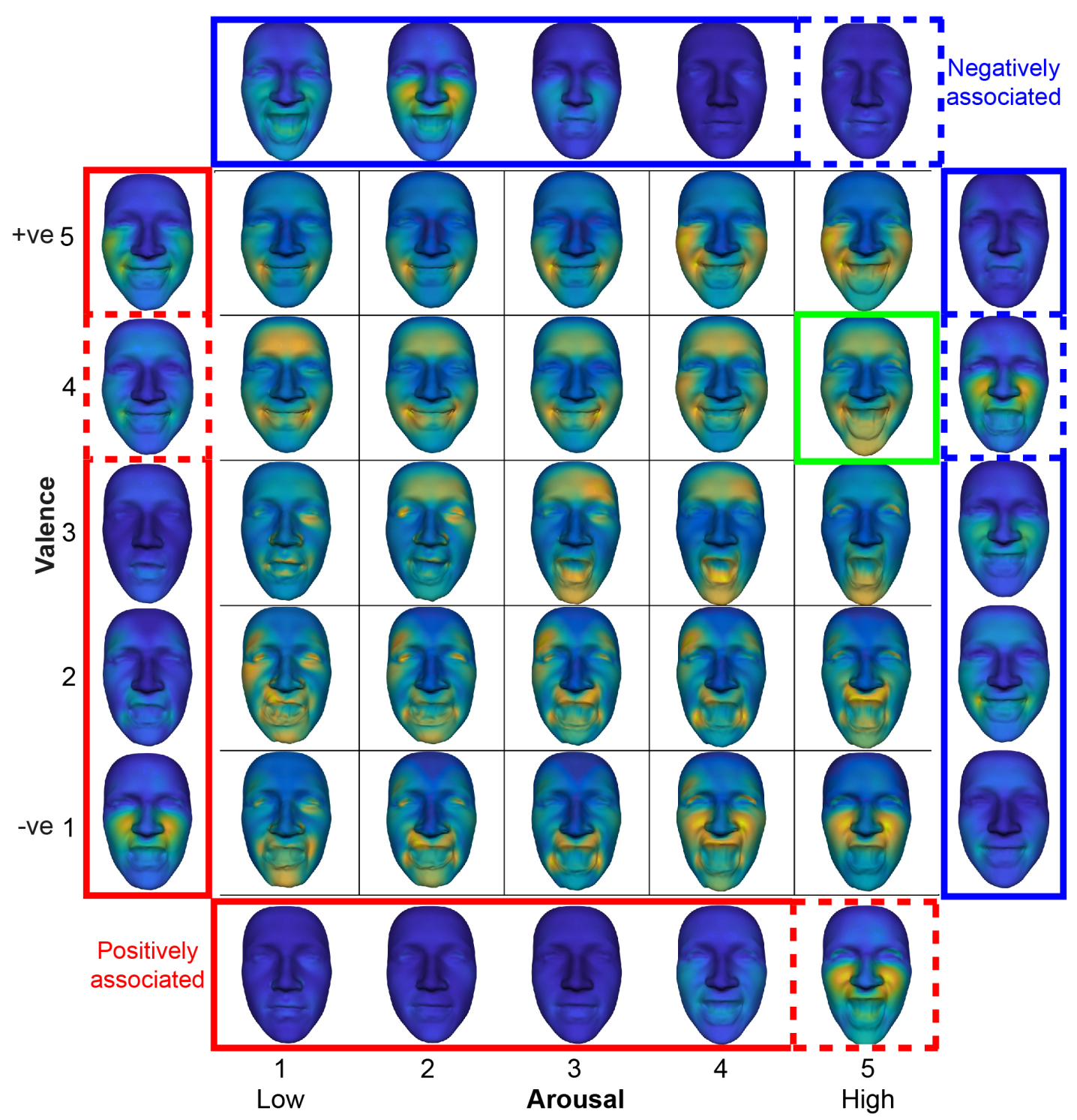

B. Combining Face Movements from Dimensions of Valence and Arousal

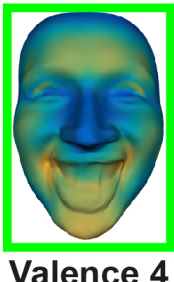

Arousal 5

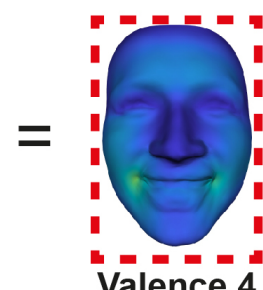

Valence 4

Positively associated

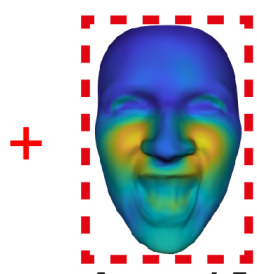

Arousal 5

Positively associated

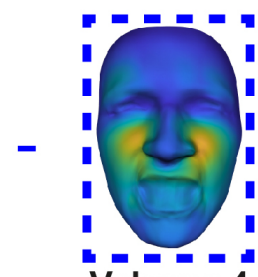

Valence 4 Negatively associated

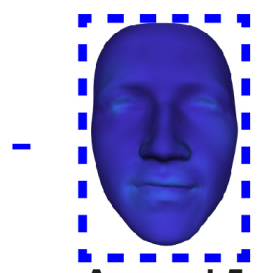

Arousal 5

Negatively associated 
Fig. S3. Building a valence-arousal space of face movements. (A) Dimensional valence-arousal space of face movements. Face maps across the 5 X 5 valence-arousal space shows the face movements associated with the perception of each level of valence and arousal rating, combined (see Fig. S2A). To build each face movement pattern - for example, outlined in green - we combined the corresponding positively associated AUs (dashed red outline) and removed any negatively associated AUs (dashed blue outline). (B) Combining face movements of valence and arousal. The example illustrated above is repeated here in linear form.

\section{Mapping facial expressions of complex emotions onto a dimensional valence-arousal space.} We tested the generalization of the results with the six classic emotions to a broader set of 19 complex emotion categories using an existing data set from Jack et al., (2016) by mapping the 19 complex emotions onto the valence-arousal space. Specifically, we computed, for each complex emotion, a distribution pattern of average correlations by correlating each of the 40 facial expression models in each cell of the valence-arousal space with all individual facial expression stimuli categorized as a given emotion (e.g., delighted). Fig. S4 shows the results for each emotion, displayed as a color-coded matrix. Warmer colors indicate positive correlations and cooler colors indicate negative correlations (see colorbar on bottom right). 


\section{Mapping of Facial Expressions of Complex Emotions}
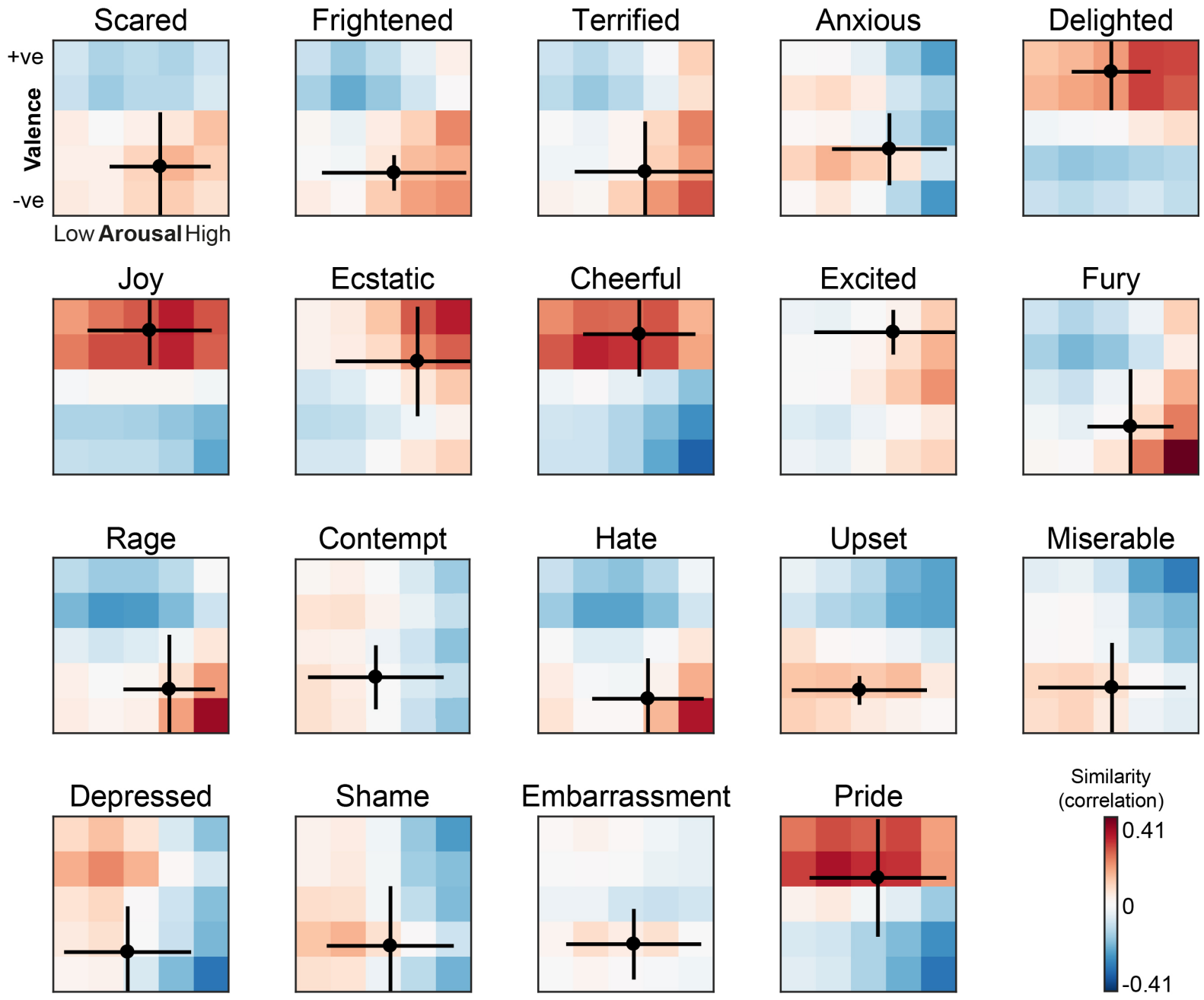

Fig. S4. Mapping facial expressions of complex emotions onto a valence-arousal space of face movements. Each color-coded matrix shows, for each of the 19 complex emotions, the average correlations between the facial expressions categorized as a given emotion (e.g., ecstatic) and the face expressions of each cell in the valence-arousal space. Warmer colors indicate positive correlations, cooler colors indicate negative correlations (see color bar on bottom right). For example, facial expressions categorized as the emotion 'ecstatic' are highly positively correlated with positive valence, high arousal cells and negatively correlated with negative, low arousal cells. The semantic location of each emotion word is represented in each matrix as a black point with crosses representing the standard deviation for each dimension. 
Composition of facial expressions of emotion categories. To evaluate the composition of face movements in the facial expression models of emotions ( 6 classic +19 complex $)$, we first established the association of each AU with valence and arousal using Pointwise Mutual Information with a permutation test applied to data pooled across participants (see in main text, Methods, Facial expression modelling procedure, dimensional-valence arousal space). Figure S5 shows the results of the PMI analysis.
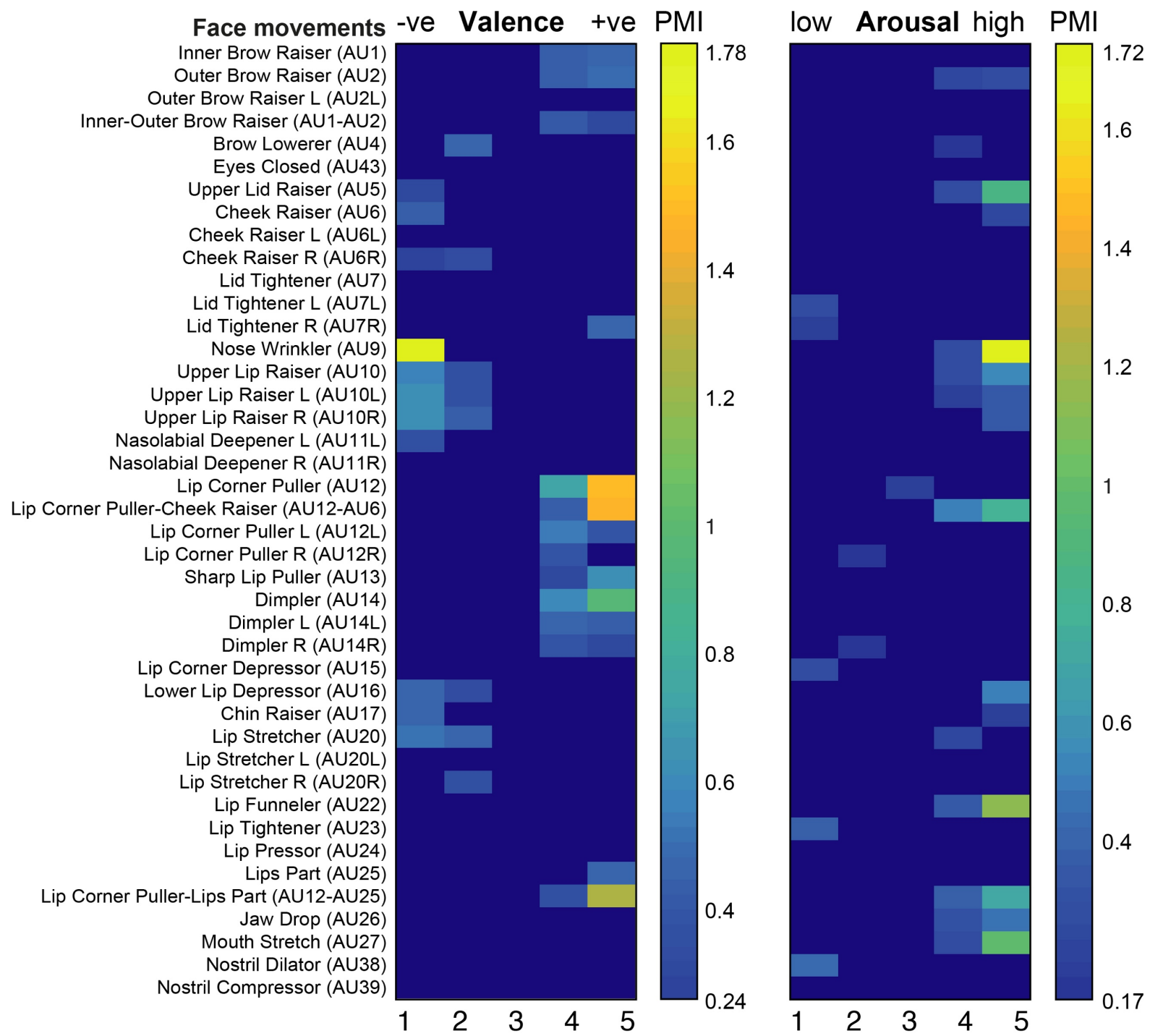

Fig. S5 Association of Action Units with valence and arousal. Each color-coded matrix shows the Pointwise Mutual Information (PMI) between each AU (when present on trials) and the participants' responses of each level of valence (left panel) and each level of arousal (right panel). Warmer colors indicate high PMI, cooler colors indicate low PMI (see colorbar on right). Only PMI values reaching statistical significance, established using a one-tailed permutation test, are shown. 
We then decomposed each facial expression model into its constituent valence and arousal AUs. Fig. S6 shows the results for the six classic emotions and 19 complex emotions as separate colorcoded matrices where color-coding represents the association of the AU with valence and arousal (see key to bottom).

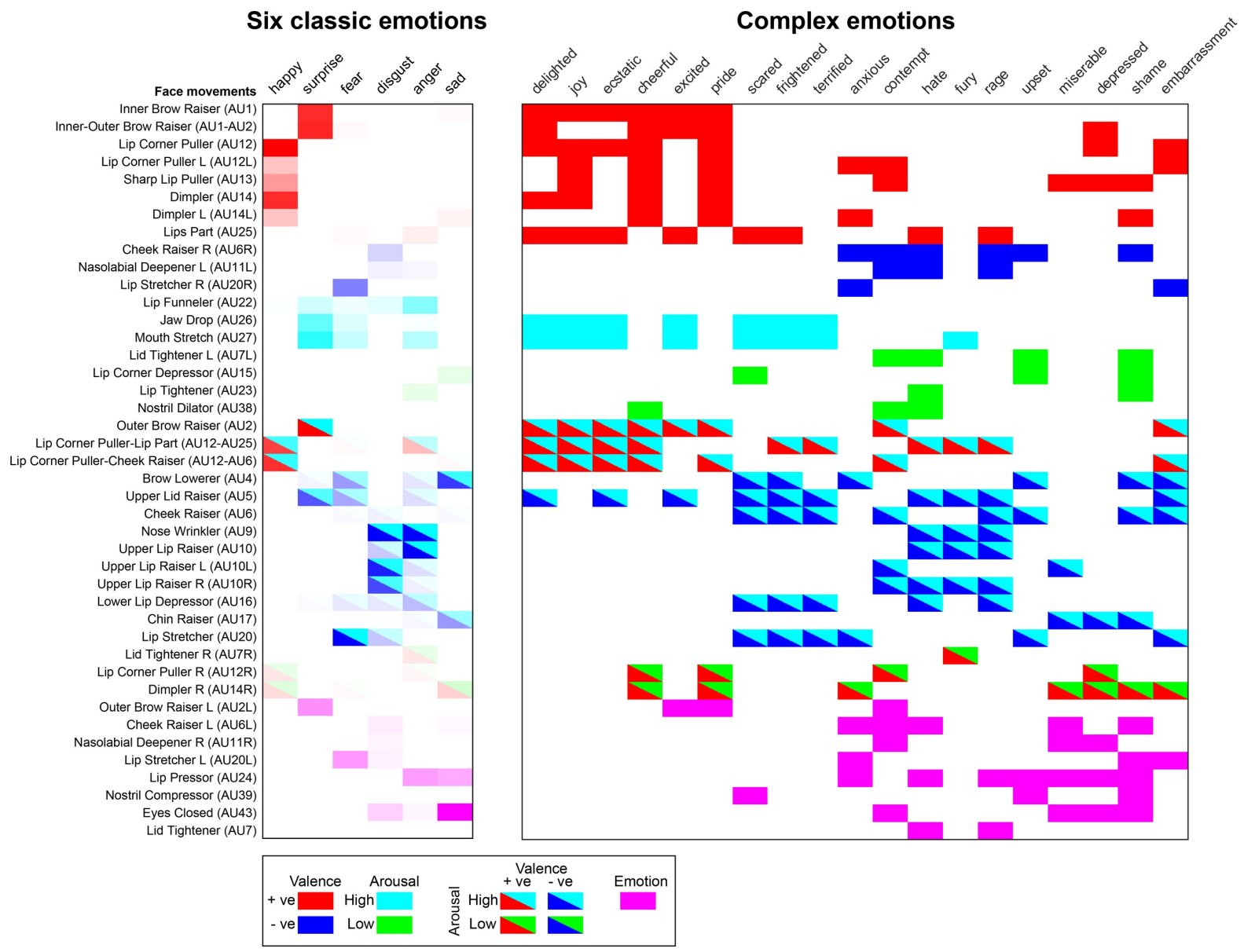

Fig. S6 Composition of facial expressions of emotion by constituent valence and arousal face movements. Each color-coded matrix shows, for the six classic and 19 complex emotions separately, the composition of the facial expression models according to their constituent valence and arousal AUs (see key to bottom). Single colors indicate the association with one affective dimension (e.g., positive valence, shown in red); two colors indicate two affective dimensions (e.g., positive valence, low arousal, shown in red/green). For the six classic emotions (left panel), results are shown summed across the 60 models; color saturation represent the proportion of models (higher saturation indicates higher proportions). For the 19 complex emotions (right 
panel), results are shown based on a model per emotion category computed across participants (data extracted from Jack et al., 2016).

Screening questionnaire. Each potential participant completed the following questionnaires. We selected only those who answered 'no' to all questions for participation in the experiment.

1. Have you ever lived in non-Western* country before (e.g. on a gap year, summer work, move due parental employment)?

2. How many weeks have you spent in a non-Western country (e.g. on vacation)?

3. Have you ever dated or had a very close friendship with a non-Western person?

4. Have you ever been involved with any non-Western culture societies/groups?

*by Western groups/countries, we are referring to Europe (East and West), USA, Canada, United Kingdom, Australia and New Zealand.

\section{SI References}

Bouma, G. (2009). Normalized (pointwise) mutual information in collocation extraction. Proceedings of GSCL, 31-40.

Jack, R. E., Sun, W., Delis, I., Garrod, O. G. B., \& Schyns, P. G. (2016). Four not six: Revealing culturally common facial expressions of emotion. Journal of Experimental Psychology: General, 145(6), 708-730. https://doi.org/10.1037/xge0000162

Nichols, T. E., \& Holmes, A. P. (2002). Nonparametric permutation tests for functional neuroimaging: A primer with examples. Human Brain Mapping, 15(1), 1-25. https://doi.org/10/fsf2fh 